\title{
Hulthén and Coulomb-Like Potentials as a Tensor Interaction within the Relativistic Symmetries of the Manning-Rosen Potential
}

\author{
Hadi Tokmehdashi, ${ }^{1}$ Ali Akbar Rajabi, ${ }^{1}$ and Majid Hamzavi ${ }^{2}$ \\ ${ }^{1}$ Department of Physics, Shahrood University of Technology, Shahrood, Iran \\ ${ }^{2}$ Department of Physics, University of Zanjan, Zanjan, Iran \\ Correspondence should be addressed to Majid Hamzavi; majid.hamzavi@gmail.com
}

Received 13 August 2013; Accepted 30 January 2014; Published 20 March 2014

Academic Editor: Chao-Qiang Geng

Copyright ( $\odot 2014$ Hadi Tokmehdashi et al. This is an open access article distributed under the Creative Commons Attribution License, which permits unrestricted use, distribution, and reproduction in any medium, provided the original work is properly cited. The publication of this article was funded by SCOAP ${ }^{3}$.

\begin{abstract}
The bound-state solutions of the Dirac equation for the Manning-Rosen potential are presented approximately for arbitrary spinorbit quantum number $\kappa$ with the Hulthén and Coulomb-like potentials as a tensor interaction. The generalized parametric Nikiforov-Uvarov (NU) method is used to obtain energy eigenvalues and corresponding two-component spinors of the two Dirac particles and these are obtained in the closed form by using the framework of the spin symmetry and p-spin symmetry concept. We have also shown that tensor interaction removes degeneracies between spin and p-spin doublets. Some numerical results are also given.
\end{abstract}

\section{Introduction}

The spin and pseudospin symmetry concepts introduced in nuclear theory $[1,2]$ have been used to explain the features of deformed nuclei [3] and superdeformation [4] and to establish an effective shell-model coupling scheme [5]. Within the framework of the relativistic mean field theory, Ginocchio $[6,7]$ has found that a Dirac Hamiltonian with scalar and vector harmonic oscillator potentials in the case of $(V(r)-S(r)=0)$ possesses not only a spin symmetry but also a $U(3)$ symmetry, but a Dirac Hamiltonian in the case of $(V(r)+S(r)=0)$ possesses a pseudospin symmetry and a pseudo-U(3) symmetry. Meng et al. [8] have showed that the pseudospin symmetry is exact under the condition $(d(V(r)+S(r)) / d r=0)$. In addition, Alhaidari et al. [9] have investigated in detail physical interpretation on the threedimensional Dirac equation in the case of spin symmetry limit $(V(r)-S(r)=0)$ and pseudospin symmetry limit $(V(r)+$ $S(r)=0)$. In recent years, by considering the importance of spin and pseudospin symmetries, some authors have contributed many works in this field. For more review of this, one can read the recent works by Wei and Dong [10-13].
The p-spin symmetry refers to a quasidegeneracy of single nucleon doublets with nonrelativistic quantum number $(n, l, j=l+1 / 2)$ and $(n-1, l+2, j=l+3 / 2)$, where $n, l$, and $j$ are single nucleon radial, orbital, and total angular quantum numbers, respectively $[1,2]$. The total angular momentum $j=\widetilde{l}+\widetilde{s}$ with $\widetilde{l}=l+1$ is a pseudoangular momentum and $\widetilde{s}$ is p-spin angular momentum [14-18].

Motivated by recent work about the spin symmetry and pseudospin symmetry solutions of the Dirac equation for arbitrary spin-orbit coupling quantum number $\kappa$ with the Eckart potential and Pöschl-Teller potential carried out by Jia et al. $[19,20]$, in this work we attempt to study the spin symmetry and pseudospin symmetry solutions of the Dirac equation for arbitrary quantum number $\mathcal{\kappa}$ with the ManningRosen potential:

$$
\begin{array}{r}
V(r)=\frac{1}{K b^{2}}\left[-\frac{A e^{-r / b}}{1-e^{-r / b}}+\frac{\alpha(\alpha-1) e^{-2 r / b}}{\left(1-e^{-r / b}\right)^{2}}\right], \\
K=\frac{2 M}{\hbar^{2}},
\end{array}
$$


where $\alpha$ and $A$ are two dimensionless parameters and $b$ is the screening parameter which is used for determining the range of potential and has dimension of length [21]. It is shown that this potential remains invariant by mapping $\alpha \leftrightarrow$ $1-\alpha$. The $s$-wave scattering solutions and bound state energy eigenvalues for the Manning-Rosen potential have been calculated by using the function analysis method [22] and path integral approach [23], respectively. Recently, Ikhdair and Sever have also studied the arbitrary $l$-wave solutions of the $D$-dimensional Schrödinger equation with the ManningRosen potential by using an approximate to the centrifugal term [24].

For more review of the Manning-Rosen potential, one can refer to the [25-29].

Tensor potentials were introduced into the Dirac equation with the substitution $\vec{p} \rightarrow \vec{p}-\operatorname{im} \omega \beta \cdot \widehat{r} U(r)$ and a spinorbit coupling is added to the Dirac Hamiltonian [30, 31].

Recently, different kinds of potentials (e.g., Coulomb-like, Linear plus Coulomb-like, and Yukawa), as tensor couplings, have been used widely in the studies of nuclear properties. In this regard, see [32-39]. Here we study a tensor potential in the Hulthén (2) and Coulomb-like (3) form as follows:

$$
U(r)=-U_{0 H} \frac{e^{-r / b}}{1-e^{-r / b}}, \quad b=\delta^{-1}, \quad U_{0 H}=Z e^{2} \delta,
$$

where $U_{0 H}$ is the potential depth, $\delta$ is the screening range parameter, and $b$ represents the spatial range. If the potential is used for atoms, then $U_{0 H}=Z \delta$ (in the relativistic units $\hbar=c=e=1$ ), where $Z$ is identified as the atomic number. The Hulthén potential behaves like the Coulomb potential near the origin (i.e., $r \rightarrow 0$ or $r \ll b) U_{C}(r)=-Z e^{2} / r$ but decreases exponentially in the asymptotic region when $r \gg 0$, so its capacity for bound states is smaller than the Coulomb potential. The Hulthén potential is widely used for the description of the nucleon-heavy nucleus interactions (see [40] and the references therein). This potential has been applied to a number of areas such as nuclear and particle physics [41-43], atomic physics [44, 45], molecular physics $[46,47]$, and chemical physics [48].

Also, the Coulomb-like potential [49] for the tensor due to a charge $Z_{a} e$ interacting with a charge $Z_{b} e$, distributed uniformly over a sphere of radius $R_{C}$, is added:

$$
U(r)=-\frac{U_{0 C}}{r}, \quad U_{0 C}=\frac{Z_{a} Z_{b} e^{2}}{4 \pi \varepsilon_{0}}, \quad r \geq R_{C},
$$

where $R_{C}=7.78 \mathrm{fm}$ is the Coulomb radius and $Z_{b}$ and $Z_{b}$ denote the charges of the projectile $a$ and the target nuclei $b$, respectively.

These potentials have been used in several branches of physics and their discrete and continuum states have been studied by a variety of techniques such as the algebraic perturbation calculations which are based upon the dynamical group structure $\mathrm{SO}(2,1)$ [50], the formalism of supersymmetric quantum mechanics within the framework of the variational method [51], the supersymmetry and shape invariance property [52], the asymptotic iteration method $[53,54]$, the NU method [55], and the approach proposed by Biedenharn et al. for the Dirac-Coulomb problem [56, 57]. Otherwise, some authors studied relativistic and nonrelativistic equations with different potentials [58-90]. In this paper, we solve approximately the Dirac equation with the Manning-Rosen potential for the spin-orbit quantum number $\kappa$ and we obtained the energy eigenvalues and wavefunctions of Dirac particle in the fields of the scalar and vector Manning-Rosen potential including a Hulthén and Coulomb tensor interaction under consideration of spin symmetry and pseudospin symmetry case.

The organization of this paper is as follows. In Section 2, we briefly introduce the Dirac equation with the ManningRosen potential and tensor potentials with arbitrary spinorbit coupling number $\kappa$ including tensor interaction under spin and p-spin symmetric limits. Also the generalized parametric NU method is presented in this section. The energy eigenvalue equations and corresponding wave functions for Hulthén and Coulomb tensor interaction in terms of the Jacobi polynomials are obtained in Sections 3 and 4. We also give some remark graphs and numerical results. We end with conclusion in Section 5.

\section{Dirac Equation Including Tensor Coupling}

The Dirac equation for fermionic massive spin- $1 / 2$ particles moving in attractive scalar $S(r)$, repulsive vector $V(r)$, and tensor $U(r)$ potentials is (in units $\hbar=c=1$ )

$$
\begin{gathered}
{[\vec{\alpha} \cdot \vec{p}+\beta(M+S(r))-i \beta \vec{\alpha} \cdot \widehat{r} U(r)] \psi(\vec{r})} \\
=[E-V(r)] \psi(\vec{r}),
\end{gathered}
$$

where $E$ is the relativistic energy of the system, $\vec{p}=-i \vec{\nabla}$ is the three-dimensional momentum operator, and $M$ is the mass of the fermionic particle. Further, $\vec{\alpha}$ and $\beta$ are the $4 \times 4$ Dirac matrices given by

$$
\vec{\alpha}=\left(\begin{array}{cc}
0 & \vec{\sigma} \\
\vec{\sigma} & 0
\end{array}\right), \quad \beta=\left(\begin{array}{cc}
I & 0 \\
0 & -I
\end{array}\right),
$$

where $I$ is $2 \times 2$ unitary matrix and $\vec{\sigma}$ are three-vector spin matrices

$$
\sigma_{1}=\left(\begin{array}{cc}
0 & 1 \\
1 & 0
\end{array}\right), \quad \sigma_{2}=\left(\begin{array}{cc}
0 & -i \\
i & 0
\end{array}\right), \quad \sigma_{3}=\left(\begin{array}{cc}
1 & 0 \\
0 & -1
\end{array}\right) .
$$

The total angular momentum operator $\vec{J}$ and spin-orbit $K=$ $(\vec{\sigma} \cdot \vec{L}+1)$, where $\vec{L}$ is orbital angular momentum of the spherical nucleons, commute with the Dirac Hamiltonian. The eigenvalues of spin-orbit coupling operator are $\kappa=(j+$ $1 / 2)>0$ and $\kappa=-(j+1 / 2)<0$ for unaligned spin $j=l-1 / 2$ and the aligned spin $j=l+1 / 2$, respectively. $\left(H^{2}, K, J^{2}, J_{z}\right)$ can be taken as the complete set of the conservative quantities. Thus, the spinor wave functions can be classified according 
to their angular momentum $j$; spin-orbit quantum number $\kappa$ and the radial quantum number $n$ can be written as follows:

$$
\psi_{n \kappa}(\vec{r})=\left(\begin{array}{c}
f_{n \kappa}(\vec{r}) \\
g_{n \kappa}(\vec{r})
\end{array}\right)=\left(\begin{array}{c}
\frac{F_{n \kappa}(r)}{r} Y_{j m}^{l}(\theta, \varphi) \\
i \frac{G_{n \kappa}(r)}{r} Y_{j m}^{\tilde{l}}(\theta, \varphi)
\end{array}\right),
$$

where $f_{n \kappa}(\vec{r})$ is the upper (large) component and $g_{n \kappa}(\vec{r})$ is the lower (small) component of the Dirac spinors. $Y_{j m}^{l}(\theta, \varphi)$ and $Y_{j m}^{\tilde{l}}(\theta, \varphi)$ are spin and $\mathrm{p}$-spin spherical harmonics, respectively, and $m$ is the projection of the angular momentum on the $z$-axis. Substituting (7) into (4) and using the following relations:

$$
\begin{gathered}
(\vec{\sigma} \cdot \vec{A})(\vec{\sigma} \cdot \vec{B})=\vec{A} \cdot \vec{B}+i \vec{\sigma} \cdot(\vec{A} \times \vec{B}), \\
(\vec{\sigma} \cdot \vec{P})=\vec{\sigma} \cdot \widehat{r}\left(\hat{r} \cdot \vec{P}+i \frac{\vec{\sigma} \cdot \vec{L}}{r}\right)
\end{gathered}
$$

together with the following properties:

$$
\begin{gathered}
(\vec{\sigma} \cdot \vec{L}) Y_{j m}^{\tilde{l}}(\theta, \phi)=(\kappa-1) Y_{j m}^{\tilde{l}}(\theta, \phi), \\
(\vec{\sigma} \cdot \vec{L}) Y_{j m}^{l}(\theta, \phi)=-(\kappa-1) Y_{j m}^{l}(\theta, \phi), \\
(\vec{\sigma} \cdot \hat{r}) Y_{j m}^{\tilde{l}}(\theta, \phi)=-Y_{j m}^{l}(\theta, \phi), \\
(\vec{\sigma} \cdot \hat{r}) Y_{j m}^{l}(\theta, \phi)=-Y_{j m}^{\tilde{l}}(\theta, \phi),
\end{gathered}
$$

one obtains two coupled differential equations for upper and lower radial wave functions $F_{n \kappa}(r)$ and $G_{n \kappa}(r)$ as

$$
\begin{aligned}
& \left(\frac{d}{d r}+\frac{\kappa}{r}-U(r)\right) F_{n \kappa}(r)=\left(M+E_{n \kappa}-\Delta(r)\right) G_{n \kappa}(r), \\
& \left(\frac{d}{d r}-\frac{\kappa}{r}+U(r)\right) G_{n \kappa}(r)=\left(M-E_{n \kappa}+\Sigma(r)\right) F_{n \kappa}(r),
\end{aligned}
$$

where

$$
\begin{aligned}
& \Delta(r)=V(r)-S(r), \\
& \Sigma(r)=V(r)+S(r) .
\end{aligned}
$$

Eliminating $F_{n \kappa}(r)$ and $G_{n \kappa}(r)$ from (10) and (11), we finally obtain the following two Schrödinger-like differential equations for the upper and lower radial spinor components, respectively:

$$
\begin{gathered}
{\left[\frac{d^{2}}{d r^{2}}-\frac{\kappa(\kappa+1)}{r^{2}}+\frac{2 \kappa}{r} U(r)-\frac{d U(r)}{d r}-U^{2}(r)\right] F_{n \kappa}(r)} \\
\quad+\frac{d \Delta(r) / d r}{M+E_{n \kappa}-\Delta(r)}\left(\frac{d}{d r}+\frac{\kappa}{r}-U(r)\right) F_{n \kappa}(r) \\
=\left[\left(M+E_{n \kappa}-\Delta(r)\right)\left(M-E_{n \kappa}+\sum(r)\right)\right] F_{n \kappa}(r), \\
{\left[\frac{d^{2}}{d r^{2}}-\frac{\kappa(\kappa-1)}{r^{2}}+\frac{2 \kappa}{r} U(r)+\frac{d U(r)}{d r}-U^{2}(r)\right] G_{n \kappa}(r)} \\
\quad+\frac{d \Sigma(r) / d r}{M-E_{n \kappa}+\Sigma(r)}\left(\frac{d}{d r}-\frac{\kappa}{r}+U(r)\right) G_{n \kappa}(r) \\
=\left[\left(M+E_{n \kappa}-\Delta(r)\right)\left(M-E_{n \kappa}+\sum(r)\right)\right] G_{n \kappa}(r),
\end{gathered}
$$

where $\kappa(\kappa-1)=\widetilde{l}(\widetilde{l}+1)$ and $\kappa(\kappa+1)=l(l+1)$.

The quantum number $\kappa$ is related to the quantum numbers for spin symmetry $l$ and p-spin symmetry $\widetilde{l}$ as

$$
\kappa= \begin{cases}-(l+1)=-\left(j+\frac{1}{2}\right), & \left(s_{1 / 2}, p_{3 / 2}, \text { etc. }\right) j=l+\frac{1}{2}, \\
& \text { aligned spin }(\kappa<0), \\
+l=+\left(j+\frac{1}{2}\right), & \left(p_{1 / 2}, d_{3 / 2}, \text { etc. }\right) j=l-\frac{1}{2}, \\
& \text { unaligned spin }(\kappa>0),\end{cases}
$$

and the quasidegenerate doublet structure can be expressed in terms of a p-spin angular momentum $\widetilde{s}=1 / 2$ and pseudoorbital angular momentum $\widetilde{l}$, which can be defined as

$$
\kappa= \begin{cases}-\widetilde{l}=-\left(j+\frac{1}{2}\right), & \left(s_{1 / 2}, p_{3 / 2}, \text { etc. }\right) j=\widetilde{l}-\frac{1}{2}, \\
& \text { alinged p-spin }(\kappa<0), \\
+(\widetilde{l}+1)=+\left(j+\frac{1}{2}\right), & \left(d_{3 / 2}, f_{5 / 2}, \text { etc. }\right) j=\widetilde{l}+\frac{1}{2}, \\
& \text { unaligned p-spin }(\kappa>0),\end{cases}
$$

where $\kappa= \pm 1, \pm 2, \ldots$. For example, $\left(1 s_{1 / 2}, 0 d_{3 / 2}\right)$ and $\left(1 p_{3 / 2}\right.$, $0 f_{5 / 2}$ ) can be considered as p-spin doublets.

2.1. Spin Symmetry Limit. In this section, we will solve Dirac equation under spin symmetry limit with ManningRosen potential and Hulthén potential as a tensor interaction. The exact spin symmetry occurs in Dirac equation when 
$d \Delta / d r=d\left(V_{V}-V_{S}\right) / d r=0$ or $\Delta=C_{s}=$ constant [91-94]. We are taking $\Sigma(r)$ and tensor $U(r)$ potentials as

$$
\begin{gathered}
\Sigma(r)=V_{0} \frac{e^{-\delta r}}{1-e^{-\delta b}}+V_{1} \frac{e^{-2 \delta r}}{\left(1-e^{-\delta r}\right)^{2}}, \\
U(r)=-U_{0 H} \frac{e^{-\delta r}}{1-e^{-\delta r}},
\end{gathered}
$$

where $\delta=1 / b, V_{0}=-A / K b^{2}$, and $V_{1}=\alpha(\alpha-1) / K b^{2}$.

In Figure 1, structure of the Manning-Rosen potential for various values of $\alpha$ screening parameter and $r$ is shown.

Under this symmetry, from (13), one obtains

$$
\begin{aligned}
& {\left[\frac{d^{2}}{d r^{2}}-\frac{\kappa(\kappa+1)}{r^{2}}-2 \kappa U_{0 H} \frac{e^{-\delta r}}{r\left(1-e^{-\delta r}\right)}\right.} \\
& \quad-U_{0 H} \delta\left(\frac{e^{-\delta r}}{1-e^{-\delta r}}+\frac{e^{-2 \delta r}}{\left(1-e^{-\delta r}\right)^{2}}\right) \\
& -U_{0 H}^{2} \frac{e^{-2 \delta r}}{\left(1-e^{-\delta r}\right)^{2}}-\gamma\left(V_{0} \frac{e^{-\delta r}}{1-e^{-\delta b}}+V_{1} \frac{e^{-2 \delta r}}{\left(1-e^{-\delta r}\right)^{2}}\right) \\
& \left.-\beta^{2}\right] F_{n \kappa}(r)=0,
\end{aligned}
$$

where $\gamma=M+E_{n \kappa}-C_{s}$ and $\beta^{2}=\left(M-E_{n \kappa}\right)\left(M+E_{n \kappa}-C_{s}\right)$. Also, $\kappa=l$ and $\kappa=-l-1$ for $\kappa<0$ and $\kappa>0$, respectively.

2.2. p-Spin Symmetry Limit. Within the pseudospin symmetry case, $d[V(r)+S(r)] / d r=d \Sigma(r) / d r=0$ or $\Sigma(r)=C_{\mathrm{ps}}=$ constant, and p-spin symmetry is exact in the Dirac equation $[8,95-97]$. In this part, we consider $\Delta(r)$ as

$$
\Delta(r)=V_{0} \frac{e^{-\delta r}}{1-e^{-\delta b}}+V_{1} \frac{e^{-2 \delta r}}{\left(1-e^{-\delta r}\right)^{2}}
$$

and tensor $U(r)$ potential as

$$
U(r)=-U_{0 H} \frac{e^{-\delta r}}{1-e^{-\delta r}} .
$$

Under this symmetry, from (14), we obtain

$$
\begin{aligned}
& {\left[\frac{d^{2}}{d r^{2}}-\frac{\kappa(\kappa-1)}{r^{2}}-2 \kappa U_{0 H} \frac{e^{-\delta r}}{r\left(1-e^{-\delta r}\right)}\right.} \\
& \quad+U_{0 H} \delta\left(\frac{e^{-\delta r}}{1-e^{-\delta r}}+\frac{e^{-2 \delta r}}{\left(1-e^{-\delta r}\right)^{2}}\right) \\
& \quad-U_{0 H}^{2} \frac{e^{-2 \delta r}}{\left(1-e^{-\delta r}\right)^{2}}-\widetilde{\gamma}\left(V_{0} \frac{e^{-\delta r}}{1-e^{-\delta b}}+V_{1} \frac{e^{-2 \delta r}}{\left(1-e^{-\delta r}\right)^{2}}\right) \\
& \left.-\widetilde{\beta}^{2}\right] G_{n \kappa}(r)=0,
\end{aligned}
$$

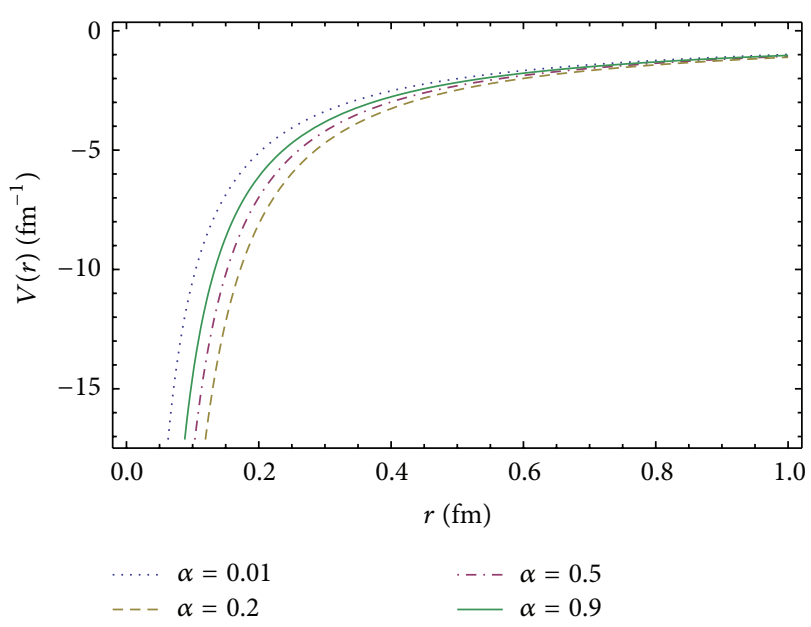

FIgURE 1: The variation of the Manning-Rosen potential according to various values of $\alpha$ screening parameter and $(\delta=1 / b=0.025$, $A=2 b, K=2$ ).

where $\tilde{\gamma}=E_{n \kappa}-M-C_{\mathrm{ps}}$ and $\widetilde{\beta}^{2}=\left(M+E_{n \kappa}\right)\left(M-E_{n \kappa}+C_{\mathrm{ps}}\right)$. Also, $\kappa=-\widetilde{l}$ and $\kappa=\widetilde{l}+1$ for $\kappa<0$ and $\kappa>0$, respectively.

The Dirac equation for the Manning-Rosen potential given by (18) and (21) cannot be solved analytically. To obtain analytical approximate solutions for the Manning-Rosen type potential with spin-orbit coupling term, we have to use an approximation in order to deal with the spin-orbit coupling term [57] as

$$
\frac{1}{r} \approx \delta \frac{e^{-\delta r}}{1-e^{-\delta r}}
$$

Or equivalently

$$
\frac{1}{r^{2}} \approx \delta^{2} \frac{e^{-2 \delta r}}{\left(1-e^{-\delta r}\right)^{2}},
$$

which is only valid for small values of the parameter $\delta$.

2.3. Basic Equations of Parametric Nikiforov-Uvarov (NU) Method. The NU method is a powerful mathematical tool that recently has been used widely to solve second-order differential equations $[98,99]$. In this section, we briefly describe this method. This technique is based on solving the hypergeometric type second-order differential equations by means of the special orthogonal functions [100]. For a given potential, the Schrödinger or Schrödinger-like equations in spherical coordinates are reduced to a generalized equation of hypergeometric type with an appropriate coordinate transformation $r \rightarrow s$ and then they are solved systematically to find the exact or particular solutions. The main equation which is closely associated with the method is given in the following form [101, 102]:

$$
\psi_{n}^{\prime \prime}(s)+\frac{\tilde{\tau}(s)}{\sigma(s)} \psi_{n}^{\prime}(s)+\frac{\widetilde{\sigma}(s)}{\sigma^{2}(s)} \psi_{n}(s)=0,
$$

where $\sigma(s)$ and $\widetilde{\sigma}(s)$ are polynomials, at most of second degree, and $\tilde{\tau}(s)$ is a first-degree polynomial. To make 
the application of the NU method simpler and direct without need to check the validity of solution, we present a shortcut for the method. Hence, firstly we write the general form of the Schrödinger-like equation (24) in a more general form as

$$
\psi_{n}^{\prime \prime}(s)+\left(\frac{c_{1}-c_{2} s}{s\left(1-c_{3} s\right)}\right) \psi_{n}^{\prime}(s)+\left(\frac{-\xi_{2} s^{2}+\xi_{1} s-\xi_{0}}{s^{2}\left(1-c_{3} s\right)^{2}}\right) \psi_{n}(s)=0
$$

satisfying the wave functions

$$
\psi_{n}(s)=\phi(s) y_{n}(s)
$$

Secondly, we compare (27) with its counterpart equation (26) to obtain the following parameter values:

$$
\begin{gathered}
\tilde{\tau}(s)=c_{1}-c_{2} s, \quad \sigma(s)=s\left(1-c_{3} s\right), \\
\tilde{\sigma}(s)=-\xi_{2} s^{2}+\xi_{1} s-\xi_{0} .
\end{gathered}
$$

Now, following the NU method [101], we obtain the energy equation $[102,103]$

$$
\begin{aligned}
c_{2} n- & (2 n+1) c_{5}+(2 n+1)\left(\sqrt{c_{9}}-c_{3} \sqrt{c_{8}}\right) \\
& +n(n-1) c_{3}+c_{7}+2 c_{3} c_{8}-2 \sqrt{c_{8} c_{9}}=0 .
\end{aligned}
$$

The values for the parametric constants are shown in Table 1.

Where $c_{12}>0, c_{13}>0$ and $s \in\left[0,1 / c_{3}\right], c_{3} \neq 0$, the corresponding wave functions are

$$
\begin{array}{r}
\rho(s)=s^{c_{10}}\left(1-c_{3} s\right)^{c_{11}}, \quad \phi(s)=s^{c_{12}}\left(1-c_{3} s\right)^{c_{13}}, \\
c_{12}>0, c_{13}>0, \\
y_{n}(s)=P_{n}^{\left(c_{10}, c_{11}\right)}\left(1-2 c_{3} s\right), \quad c_{10}>-1, c_{11}>-1, \\
\psi_{n \kappa}(s)=N_{n \kappa} s^{c_{12}}\left(1-c_{3} s\right)^{c_{13}} P_{n}^{\left(c_{10}, c_{11}\right)}\left(1-2 c_{3} s\right),
\end{array}
$$

where $P_{n}^{(\mu, v)}(x), \mu>-1, \nu>-1$, and $x \in[-1,1]$ are Jacobi polynomials.

\section{Bound States of the Manning-Rosen Potential with Hulthén Tensor Interaction}

3.1. Spin Symmetry Solution. Substituting (22) and (23) into (18) gives

$$
\begin{aligned}
& {\left[\frac{d^{2}}{d r^{2}}-\left\{\kappa(\kappa+1) \delta^{2}+(2 \kappa+1) U_{0 H} \delta+U_{0 H}^{2}+\gamma V_{1}\right\}\right.} \\
& \quad \times \frac{e^{-2 \delta r}}{\left(1-e^{-\delta r}\right)^{2}}+\left\{-U_{0 H} \delta-\gamma V_{0}\right\} \\
& \left.\quad \times \frac{e^{-\delta r}}{1-e^{-\delta b}}-\beta^{2}\right] F_{n \kappa}(r)=0 .
\end{aligned}
$$

Defining a new variable of the form $s=e^{-\delta r}$ and using (30), we obtain

$$
\begin{aligned}
& \frac{d^{2} F_{n \kappa}(s)}{d s^{2}}+\frac{1-s}{s(1-s)} \frac{d F_{n \kappa}(s)}{d s}+\frac{1}{\delta^{2} s^{2}(1-s)^{2}} \\
& \times\left[-\left(A+B+\beta^{2}\right) s^{2}+\left(B+2 \beta^{2}\right) s-\beta^{2}\right] \\
& \times F_{n \kappa}(s)=0,
\end{aligned}
$$

where

$$
\begin{gathered}
A=\kappa(\kappa+1) \delta^{2}+(2 \kappa+1) U_{0 H} \delta+U_{0 H}^{2}+\gamma V_{1}, \\
B=-U_{0 H} \delta-\gamma V_{0} .
\end{gathered}
$$

Comparing (31) and (25), we can easily obtain the coefficients $c_{i}(i=1,2,3)$ and analytical expressions $\xi_{j}(j=0,1,2)$. By using Table 1 , we are obtained the coefficients $c_{i}(i=$ $4,5, \ldots, 13)$. The values of all coefficients are shown in Table 2.

In Table 2, $\eta_{\kappa}=\left((\kappa+1 / 2)^{2}+\left(U_{0 H} / \delta\right)\left(\left(U_{0 H} / \delta\right)+\right.\right.$ $\left.2 \kappa+1)+\gamma V_{1} / \delta^{2}\right)^{1 / 2}$, and by using (28), we can obtain the energy eigenvalues of the Manning-Rosen potential with spin symmetric limit as

$$
\begin{aligned}
& \left(n+\frac{1}{2}+\sqrt{\left(\kappa+\frac{1}{2}\right)^{2}+\frac{U_{0 H}}{\delta}\left(\frac{U_{0 H}}{\delta}+2 \kappa+1\right)+\frac{\gamma V_{1}}{\delta^{2}}}\right. \\
& \left.-\sqrt{\frac{\beta^{2}}{\delta^{2}}}\right)^{2} \\
& =\frac{\gamma\left(V_{1}-V_{0}\right)+\beta^{2}}{\delta^{2}}+\left(\kappa+\frac{1}{2}\right)^{2} \\
& \quad+\frac{U_{0 H}}{\delta}\left(\frac{U_{0 H}}{\delta}+2 \kappa\right)-\frac{1}{4} .
\end{aligned}
$$

To find the corresponding wave functions, referring to Table 2 and (29), we find the functions

$$
\begin{gathered}
\rho(s)=s^{-2 \sqrt{\beta^{2} / \delta^{2}}}(1-s)^{2 \eta_{\kappa}}, \\
\phi(s)=s^{-\sqrt{\beta^{2} / \delta^{2}}}(1-s)^{\eta_{\kappa}+1 / 2}, \\
y_{n}(s)=P_{n}^{\left(-2 \sqrt{\beta^{2} / \delta^{2}}, 2 \eta_{\kappa}\right)}(1-2 s) .
\end{gathered}
$$

By using $F_{n \kappa}(s)=\phi(s) y_{n}(s)$, we get the upper component of the Dirac spinor as

$$
\begin{aligned}
F_{n \kappa}(r)= & N_{n \kappa} e^{-\sqrt{\beta^{2}} r}\left(1-e^{-\delta r}\right)^{\eta_{\kappa}+1 / 2} \\
& \times P_{n}^{\left(-2 \sqrt{\beta^{2} / \delta^{2}}, 2 \eta_{\kappa}\right)}\left(1-2 e^{-\delta r}\right),
\end{aligned}
$$

where $N_{n \kappa}$ is normalization constant. The lower component of the Dirac spinor from (10) can be calculated as

$$
G_{n \kappa}(r)=\frac{1}{M+E_{n \kappa}-C_{s}}\left(\frac{d}{d r}+\frac{\kappa}{r}-U(r)\right) F_{n \kappa}(r),
$$


TABLE 1: The values for the parametric constants of the NU method.

\begin{tabular}{|c|c|c|c|c|}
\hline \multicolumn{5}{|c|}{ Parametric NU constants } \\
\hline$\xi_{0}$ & $c_{1}$ & $c_{4}=\frac{1}{2}\left(1-c_{1}\right)$ & $c_{7}=2 c_{4} c_{5}-\xi_{1}$ & $c_{10}=c_{1}+2 c_{4}-2 \sqrt{c_{8}}-1>-1$ \\
\hline$\xi_{1}$ & $c_{2}$ & $c_{5}=\frac{1}{2}\left(c_{2}-2 c_{3}\right)$ & $c_{8}=c_{4}^{2}+\xi_{0}$ & $c_{11}=1-c_{1}-2 c_{4}+\frac{2}{c_{3}} \sqrt{c_{9}}>-1, \quad c_{3} \neq 0$ \\
\hline$\xi_{2}$ & $c_{3}$ & $c_{6}=c_{5}^{2}+\xi_{2}$ & $c_{9}=c_{3}\left(c_{7}+c_{3} c_{8}\right)+c_{6}$ & $c_{12}=c_{4}-\sqrt{c_{8}}>0$ \\
\hline & & & $c_{13}=-c_{4}+\frac{1}{c_{3}}\left(\sqrt{c_{9}}-c_{5}\right)>0, \quad c_{3} \neq 0$ & \\
\hline
\end{tabular}

TABLE 2: The specific values for the parametric constants necessary for calculating the energy eigenvalues and eigenfunctions of the spin symmetry limit.

\begin{tabular}{lccc}
\hline Constant & Analytic value & Constant & Analytic value \\
\hline$c_{1} c_{1}$ & 1 & $c_{2}$ & 1 \\
\hline$c_{3}$ & 1 & $c_{4}$ & 0 \\
\hline$c_{5}$ & $-\frac{1}{2}$ & $c_{6}$ & $\frac{1}{4}+\frac{A+B+\beta^{2}}{\delta^{2}}$ \\
\hline$c_{7}$ & $-\frac{B+2 \beta^{2}}{\delta^{2}}$ & $c_{8}$ & $\frac{\beta^{2}}{\delta^{2}}$ \\
\hline$c_{9}$ & $\frac{1}{4}+\frac{A}{\delta^{2}}$ & $c_{10}$ & $-2 \sqrt{\frac{\beta^{2}}{\delta^{2}}}$ \\
\hline$c_{11}$ & $2 \eta_{\kappa}$ & $c_{12}$ & $-\sqrt{\frac{\beta^{2}}{\delta^{2}}}$ \\
\hline$c_{13}$ & $\eta_{\kappa}+\frac{1}{2}$ & $\xi_{0}$ & $\frac{\beta^{2}}{\delta^{2}}$ \\
\hline$\xi_{1}$ & $\frac{B+2 \beta^{2}}{\delta^{2}}$ & $\xi_{2}$ & $\frac{A+B+\beta^{2}}{\delta^{2}}$ \\
\hline
\end{tabular}

where $E \neq-M+C_{s}$. Some numerical results of (33) are given in Table 3. In Table 3, we obtain numerical results in the spin symmetry in the presence and absence of tensor Hulthén form potential. We took a set of parameter values, $M=5.0 \mathrm{fm}^{-1}, C_{s}=0, \delta=0.025 \mathrm{fm}^{-1}$, and $\alpha=0.01$.

We can observe that every pair of orbitals $\left(n p_{1 / 2}, n p_{3 / 2}\right)$, $\left(n d_{3 / 2}, n d_{5 / 2}\right)$, and $\left(n f_{5 / 2}, n f_{7 / 2}\right)$ has the same energy in the absence of the tensor potential $\left(U_{0 H}=0\right)$. Thus, they can be viewed as the spin doublets; that is, the state $1 p_{1 / 2}$ with $n=1$ and $\kappa=1$ forms a spin doublet with the $1 p_{3 / 2}$ state with $n=1$ and $\kappa=-2$. On the other hand, in the presence of the tensor potential $\left(U_{0 H} \neq 0\right)$, one can notice that degeneracy between every pair of spin doublets is removed. In Figure 2, we have investigated the effect of the tensor potential on the spin doublet splitting by considering the following pairs of orbital: $\left(1 p_{3 / 2}, 1 p_{1 / 2}\right),\left(1 f_{7 / 2}, 1 f_{5 / 2}\right)$. From Figure 2, we observe that, in the case of $\left(U_{0 H}=0\right)$ (no tensor interaction), members of spin doublets have the same energy. However, in the presence of the tensor potential $\left(U_{0 H} \neq 0\right)$, these degeneracies are removed.

3.2. $p$-Spin Symmetry Solution. In this subsection we will obtain the energy eigenvalues and the corresponding wave functions for the p-spin symmetric limit of the ManningRosen potential, that is, solutions of (21). Substituting (22) and (23) into (21) gives

$$
\begin{aligned}
& {\left[\frac{d^{2}}{d r^{2}}-\left\{\kappa(\kappa-1) \delta^{2}+(2 \kappa-1) U_{0 H} \delta+U_{0 H}^{2}+\tilde{\gamma} V_{1}\right\}\right.} \\
& \quad \times \frac{e^{-2 \delta r}}{\left(1-e^{-\delta r}\right)^{2}}+\left\{U_{0 H} \delta-\tilde{\gamma} V_{0}\right\} \frac{e^{-\delta r}}{1-e^{-\delta b}} \\
& \left.-\widetilde{\beta}^{2}\right] G_{n \kappa}(r)=0,
\end{aligned}
$$

and further making the change of variables $s=e^{-\delta r}$, we obtain

$$
\begin{aligned}
& \frac{d^{2} G_{n \kappa}(s)}{d s^{2}}+\frac{1-s}{s(1-s)} \frac{d G_{n \kappa}(s)}{d s}+\frac{1}{\delta^{2} s^{2}(1-s)^{2}} \\
& \times\left[-\left(\widetilde{A}+\widetilde{B}+\widetilde{\beta}^{2}\right) s^{2}+\left(\widetilde{B}+2 \widetilde{\beta}^{2}\right) s-\widetilde{\beta}^{2}\right] \\
& \times G_{n \kappa}(s)=0,
\end{aligned}
$$


TABLE 3: The bound state energy eigenvalues in unit of $\mathrm{fm}^{-1}$ of the spin symmetry Hulthén potential for several values of $n$ and $\kappa$. Here the first and second states column according to $(l, j)$.

\begin{tabular}{|c|c|c|c|c|c|c|c|}
\hline$|n, l, \kappa\rangle$ & State & $\begin{array}{c}\alpha=0.01 \\
U_{0 H}=0 \\
E_{n, \kappa}(\kappa<0)\end{array}$ & $\begin{array}{c}\alpha=0.01 \\
U_{0 H}=0.2 \\
E_{n, \kappa}(\kappa<0)\end{array}$ & $|n, l, \kappa\rangle$ & State & $\begin{array}{c}\alpha=0.01 \\
U_{0 H}=0 \\
E_{n, \kappa}(\kappa>0)\end{array}$ & $\begin{array}{c}\alpha=0.01 \\
U_{0 H}=0.1 \\
E_{n, \kappa}(\kappa>0)\end{array}$ \\
\hline$|0,1,-2\rangle$ & $0 p_{3 / 2}$ & -4.9999843769 & -4.9999282575 & $|0,1,1\rangle$ & $0 p_{1 / 2}$ & -4.9999843769 & -4.9999566000 \\
\hline$|0,2,-3\rangle$ & $0 d_{5 / 2}$ & -4.9999843762 & -4.9999149381 & $|0,2,2\rangle$ & $0 d_{3 / 2}$ & -4.9999843762 & -4.9999614178 \\
\hline$|0,3,-4\rangle$ & $0 f_{7 / 2}$ & -4.9999843759 & -4.9998943876 & $|0,3,3\rangle$ & $0 f_{5 / 2}$ & -4.9999843759 & -4.9999648452 \\
\hline$|0,4,-5\rangle$ & $0 g_{9 / 2}$ & -4.9999843757 & -4.9998593993 & $|0,4,4\rangle$ & $0 g_{7 / 2}$ & -4.9999843757 & -4.9999674008 \\
\hline$|1,1,-2\rangle$ & $1 p_{3 / 2}$ & -4.9999149463 & -4.9997802943 & $|1,1,1\rangle$ & $1 p_{1 / 2}$ & -4.9999149463 & -4.9998313307 \\
\hline$|1,2,-3\rangle$ & $1 d_{5 / 2}$ & -4.9999023580 & -4.9997675648 & $|1,2,2\rangle$ & $1 d_{3 / 2}$ & -4.9999023580 & -4.9998349749 \\
\hline$|1,3,-4\rangle$ & $1 f_{7 / 2}$ & -4.9998943876 & -4.9997500354 & $|1,3,3\rangle$ & $1 f_{5 / 2}$ & -4.9998943876 & -4.9998377819 \\
\hline$|1,4,-5\rangle$ & $1 g_{9 / 2}$ & -4.9998888999 & -4.9997244252 & $|1,4,4\rangle$ & $1 g_{7 / 2}$ & -4.9998888999 & -4.9998400102 \\
\hline
\end{tabular}

where

$$
\begin{gathered}
\widetilde{A}=\kappa(\kappa-1) \delta^{2}+(2 \kappa-1) U_{0 H} \delta+U_{0 H}^{2}+\widetilde{\gamma} V_{1} \\
\widetilde{B}=U_{0 H} \delta-\widetilde{\gamma} V_{0} .
\end{gathered}
$$

On the other hand, to avoid repetition in the solution of (38), in the p-spin symmetric case,

$$
\begin{aligned}
& \left(n+\frac{1}{2}+\sqrt{\left(\kappa-\frac{1}{2}\right)^{2}+\frac{U_{0 H}}{\delta}\left(\frac{U_{0 H}}{\delta}+2 \kappa-1\right)+\frac{\tilde{\gamma} V_{1}}{\delta^{2}}}\right. \\
& \left.-\sqrt{\frac{\widetilde{\beta}^{2}}{\delta^{2}}}\right)^{2} \\
& =\frac{\tilde{\gamma}\left(V_{1}-V_{0}\right)+\widetilde{\beta}^{2}}{\delta^{2}}+\left(\kappa-\frac{1}{2}\right)^{2} \\
& \quad+\frac{U_{0 H}}{\delta}\left(\frac{U_{0 H}}{\delta}+2 \kappa\right)-\frac{1}{4} .
\end{aligned}
$$

Also, we get the lower component of the Dirac spinor as

$$
\begin{aligned}
G_{n \kappa}(r)= & \widetilde{N}_{n \kappa} e^{-\sqrt{\widetilde{\beta}^{2}} r}\left(1-e^{-\delta r}\right)^{\widetilde{\eta}_{\kappa}+1 / 2} P_{n}^{\left(-2 \sqrt{\widetilde{\beta}^{2} / \delta^{2}}, 2 \widetilde{\eta}_{\kappa}\right)} \\
& \times\left(1-2 e^{-\delta r}\right)
\end{aligned}
$$

where $\widetilde{N}_{n \kappa}$ is normalization constant and $\tilde{\eta}_{\kappa}=$ $\sqrt{(\kappa-1 / 2)^{2}+\left(U_{0 H} / \delta\right)\left(U_{0 H} / \delta+2 \kappa-1\right)+\left(\widetilde{\gamma} V_{1}\right) / \delta^{2}}$.

The upper component of the Dirac spinor from (11) can be calculated as

$$
F_{n \kappa}(r)=\frac{1}{M-E_{n \kappa}+C_{\mathrm{ps}}}\left(\frac{d}{d r}-\frac{\kappa}{r}+U(r)\right) G_{n \kappa}(r),
$$

where $E \neq M+C_{\mathrm{ps}}$. In Table 4 , we use the same parameters as in the previous subsection (with $C_{\mathrm{ps}}=0$ ) and we obtain numerical results in the $\mathrm{p}$-spin symmetry in the presence and absence of tensor Hulthén form potential. The existence of the degeneracy was observed in the following doublets:

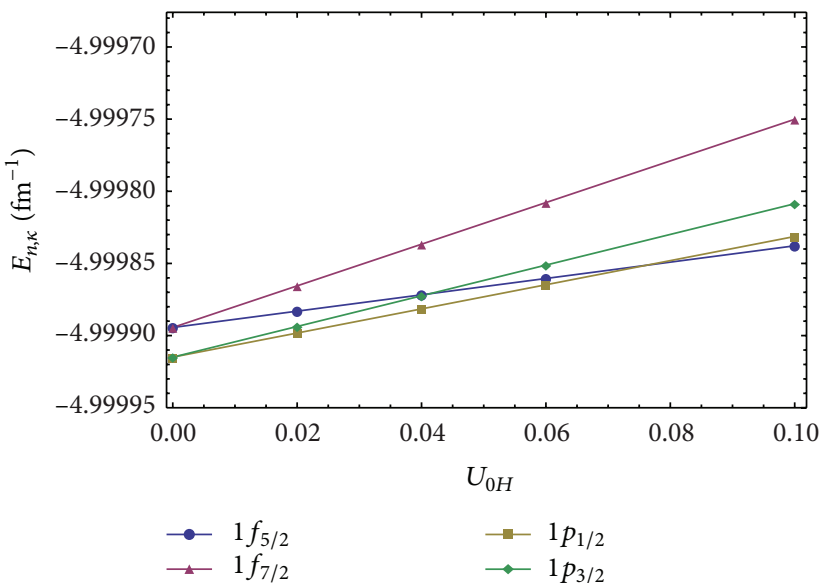

FIGURE 2: Contribution of the tensor Hulthén potential parameter to the energy levels in the case of spin symmetry.

$\left(1 s_{1 / 2}, 0 d_{3 / 2}\right),\left(1 p_{3 / 2}, 0 f_{5 / 2}\right),\left(1 d_{5 / 2}, 0 g_{7 / 2}\right),\left(1 f_{7 / 2}, 0 h_{9 / 2}\right)$, and so on, in the absence of the tensor potential $\left(U_{0 H}=0\right)$, and they are considered as p-spin doublets. In Figure 3, we have investigated the effect of the tensor potential on the p-spin doublet splitting by considering the following pairs of orbital: $\left(1 d_{5 / 2}, 0 g_{7 / 2}\right),\left(2 f_{7 / 2}, 1 h_{9 / 2}\right)$.

The energy spectrum of spin and p-spin symmetry given by Tables 3 and 4 changes with the relevant quantum numbers as well as the screening parameter $\delta$. The variation of the energy spectrum $\left(E_{n, \kappa}\right)$ according to the screening parameter $\delta$ for some states is shown in Figures 4 and 5.

\section{Bound States of the Manning-Rosen Potential with Coulomb Tensor Interaction}

In this stage we will solve Dirac (13) and (14) that are obtained in Section 2, for Manning-Rosen potential including a Coulomb-like tensor interaction under consideration of spin and pseudospin symmetry case. 
TABLE 4: The bound state energy eigenvalues in unit of $\mathrm{fm}^{-1}$ of the $\mathrm{p}$-spin symmetry Hulthén potential. Here the first states column according to $(l, j)$ and second states column $(l+2, j+1)$.

\begin{tabular}{|c|c|c|c|c|c|c|c|}
\hline$|n, \tilde{l}, \kappa\rangle$ & State & $\begin{array}{c}\alpha=0.01 \\
U_{0 H}=0 \\
E_{n, \kappa}(\kappa<0)\end{array}$ & $\begin{array}{c}\alpha=0.01 \\
U_{0 H}=0.2 \\
E_{n, \kappa}(\kappa<0)\end{array}$ & $|n, \tilde{l}, \kappa\rangle$ & State & $\begin{array}{c}\alpha=0.01 \\
U_{0 H}=0 \\
E_{n, \kappa}(\kappa>0)\end{array}$ & $\begin{array}{c}\alpha=0.01 \\
U_{0 H}=0.1 \\
E_{n, \kappa}(\kappa>0)\end{array}$ \\
\hline$|1,1,-1\rangle$ & $1 s_{1 / 2}$ & -4.9868996028 & -4.9978434057 & $|0,1,2\rangle$ & $0 d_{3 / 2}$ & -4.9868996028 & -4.9971232725 \\
\hline$|1,2,-2\rangle$ & $1 p_{3 / 2}$ & -4.9921014723 & -4.9973604254 & $|0,2,3\rangle$ & $0 f_{5 / 2}$ & -4.9921014723 & -4.9976559580 \\
\hline$|1,3,-3\rangle$ & $1 d_{5 / 2}$ & -4.9945990389 & -4.9966407919 & $|0,3,4\rangle$ & $0 g_{7 / 2}$ & -4.9945990389 & -4.9980326519 \\
\hline$|1,4,-4\rangle$ & $1 f_{7 / 2}$ & -4.9960022563 & -4.9954882731 & $|0,4,5\rangle$ & $0 h_{9 / 2}$ & -4.9960022563 & -4.9983102937 \\
\hline$|2,1,-1\rangle$ & $2 s_{1 / 2}$ & -4.9913874762 & -4.9913874762 & $|1,1,2\rangle$ & $1 d_{3 / 2}$ & -4.9913874762 & -4.9970467176 \\
\hline$|2,2,-2\rangle$ & $2 p_{3 / 2}$ & -4.9938803034 & -4.9938803034 & $|1,2,3\rangle$ & $1 f_{5 / 2}$ & -4.9938803034 & -4.9974493983 \\
\hline$|2,3,-3\rangle$ & $2 d_{5 / 2}$ & -4.9953083139 & -4.9953083139 & $|1,3,4\rangle$ & $1 g_{7 / 2}$ & -4.9953083139 & -4.9977503418 \\
\hline$|2,4,-4\rangle$ & $2 f_{7 / 2}$ & -4.9962128946 & -4.9962128946 & $|1,4,5\rangle$ & $1 h_{9 / 2}$ & -4.9962128946 & -4.9979825424 \\
\hline
\end{tabular}

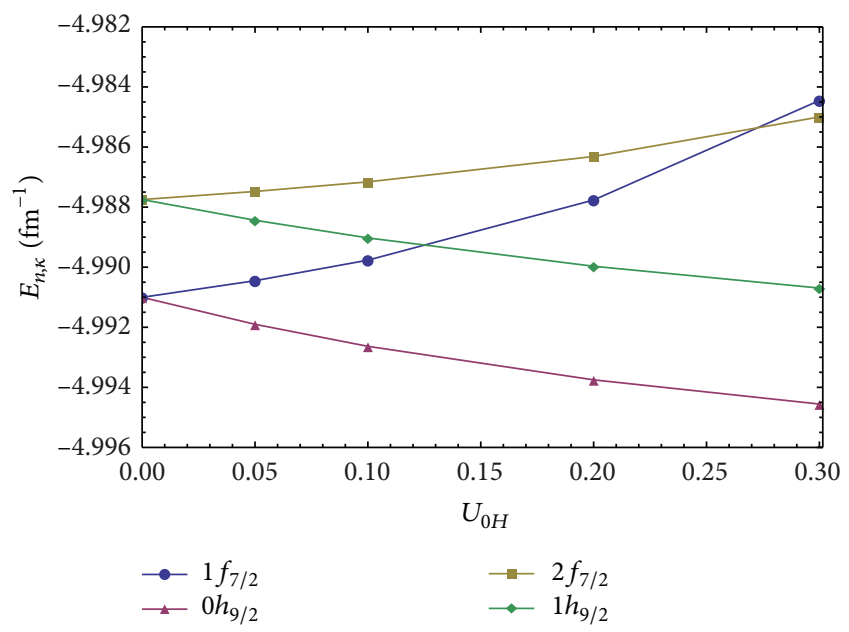

Figure 3: Contribution of the tensor Hulthén potential parameter to the energy levels in the case of p-spin symmetry.

Under spin symmetry, from (13), for the upper radial spinor component, we obtain

$$
\begin{aligned}
& {\left[\frac{d^{2}}{d r^{2}}-\frac{\lambda_{\kappa}\left(\lambda_{\kappa}+1\right)}{r^{2}}-\gamma\left(V_{0} \frac{e^{-\delta r}}{1-e^{-\delta b}}+V_{1} \frac{e^{-2 \delta r}}{\left(1-e^{-\delta r}\right)^{2}}\right)\right.} \\
& \left.-\beta^{2}\right] F_{n \kappa}(r)=0,
\end{aligned}
$$

where $\lambda_{\kappa}=\kappa+U_{0 C}, \gamma=M+E_{n \kappa}-C_{s}$, and $\beta^{2}=(M-$ $\left.E_{n \kappa}\right)\left(M+E_{n \kappa}-C_{s}\right)$. Also, $\kappa=l$ and $\kappa=-l-1$ for $\kappa<0$ and $\kappa>0$, respectively.

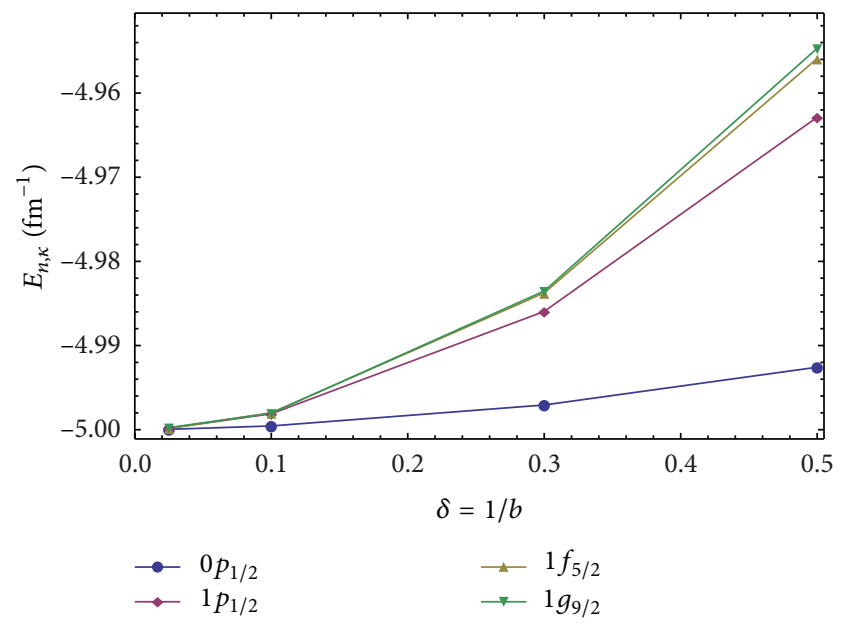

FIGURE 4: The variation of the energy spectrum $E_{n, k}$ for tensor Hulthén potential in the case of spin symmetry according to the screening parameter $\delta$. Here we selected $U_{0 H}=0.1, \alpha=0.01$.

Also under p-spin symmetry, from (14), for the lower radial spinor component, we have

$$
\begin{aligned}
& {\left[\frac{d^{2}}{d r^{2}}-\frac{\lambda_{\kappa}\left(\lambda_{\kappa}-1\right)}{r^{2}}-\tilde{\gamma}\left(V_{0} \frac{e^{-\delta r}}{1-e^{-\delta b}}+V_{1} \frac{e^{-2 \delta r}}{\left(1-e^{-\delta r}\right)^{2}}\right)\right.} \\
& \left.-\widetilde{\beta}^{2}\right] G_{n \kappa}(r)=0,
\end{aligned}
$$

where $\lambda_{\kappa}=\kappa+U_{0 C}, \tilde{\gamma}=E_{n \kappa}-M-C_{\mathrm{ps}}$, and $\widetilde{\beta}^{2}=(M+$ $\left.E_{n \kappa}\right)\left(M-E_{n \kappa}+C_{\mathrm{ps}}\right)$. Also, $\kappa=-\widetilde{l}$ and $\kappa=\widetilde{l}+1$ for $\kappa<0$ and $\kappa>0$, respectively. 
TABLE 5: The bound state energy eigenvalues in unit of $\mathrm{fm}^{-1}$ of the spin symmetry Coulomb potential for several values of $n$ and $\kappa$. Here the first and second states column according to $(l, j)$.

\begin{tabular}{|c|c|c|c|c|c|c|c|}
\hline$|n, l, \kappa\rangle$ & State & $\begin{array}{c}\alpha=0.01 \\
U_{0 C}=0 \\
E_{n, \kappa}(\kappa<0)\end{array}$ & $\begin{array}{c}\alpha=0.01 \\
U_{0 C}=1 \\
E_{n, \kappa}(\kappa<0)\end{array}$ & $|n, l, \kappa\rangle$ & State & $\begin{array}{c}\alpha=0.01 \\
U_{0 C}=0 \\
E_{n, \kappa}(\kappa>0)\end{array}$ & $\begin{array}{c}\alpha=0.01 \\
U_{0 C}=1 \\
E_{n, \kappa}(\kappa>0)\end{array}$ \\
\hline$|0,1,-2\rangle$ & $0 p_{3 / 2}$ & -4.9999843769 & -4.9999843788 & $|0,1,1\rangle$ & $0 p_{1 / 2}$ & -4.9999843769 & -4.9999843762 \\
\hline$|0,2,-3\rangle$ & $0 d_{5 / 2}$ & -4.9999843762 & -4.9999843769 & $|0,2,2\rangle$ & $0 d_{3 / 2}$ & -4.9999843762 & -4.9999843759 \\
\hline$|0,3,-4\rangle$ & $0 f_{7 / 2}$ & -4.9999843759 & -4.9999843762 & $|0,3,3\rangle$ & $0 f_{5 / 2}$ & -4.9999843759 & -4.9999843757 \\
\hline$|0,4,-5\rangle$ & $0 g_{9 / 2}$ & -4.9999843757 & -4.9999843759 & $|0,4,4\rangle$ & $0 g_{7 / 2}$ & -4.9999843757 & -4.9999843756 \\
\hline$|1,1,-2\rangle$ & $1 p_{3 / 2}$ & -4.9999149463 & -4.9999375152 & $|1,1,1\rangle$ & $1 p_{1 / 2}$ & -4.9999149463 & -4.9999023580 \\
\hline$|1,2,-3\rangle$ & $1 d_{5 / 2}$ & -4.9999023580 & -4.9999149463 & $|1,2,2\rangle$ & $1 d_{3 / 2}$ & -4.9999023580 & -4.9998943876 \\
\hline$|1,3,-4\rangle$ & $1 f_{7 / 2}$ & -4.9998943876 & -4.9999023580 & $|1,3,3\rangle$ & $1 f_{5 / 2}$ & -4.9998943876 & -4.9998888999 \\
\hline$|1,4,-5\rangle$ & $1 g_{9 / 2}$ & -4.9998888999 & -4.9998943876 & $|1,4,4\rangle$ & $1 g_{7 / 2}$ & -4.9998888999 & -4.9998848950 \\
\hline
\end{tabular}

TABLE 6: The bound state energy eigenvalues in unit of $\mathrm{fm}^{-1}$ of the $\mathrm{p}$-spin symmetry Coulomb potential. Here the first states column according to $(l, j)$ and second states column $(l+2, j+1)$.

\begin{tabular}{|c|c|c|c|c|c|c|c|}
\hline$|n, \tilde{l}, \kappa\rangle$ & State & $\begin{array}{c}\alpha=0.01 \\
U_{0 C}=0 \\
E_{n, \kappa}(\kappa<0)\end{array}$ & $\begin{array}{c}\alpha=0.01 \\
U_{0 C}=1 \\
E_{n, \kappa}(\kappa<0)\end{array}$ & $|n, \tilde{l}, \kappa\rangle$ & State & $\begin{array}{c}\alpha=0.01 \\
U_{0 C}=0 \\
E_{n, \kappa}(\kappa>0)\end{array}$ & $\begin{array}{c}\alpha=0.01 \\
U_{0 C}=1 \\
E_{n, \kappa}(\kappa>0)\end{array}$ \\
\hline$|1,1,-1\rangle$ & $1 s_{1 / 2}$ & -4.9868996028 & -4.9727532453 & $|0,1,2\rangle$ & $0 d_{3 / 2}$ & -4.9868996028 & -4.9921014723 \\
\hline$|1,2,-2\rangle$ & $1 p_{3 / 2}$ & -4.9921014723 & -4.9868996028 & $|0,2,3\rangle$ & $0 f_{5 / 2}$ & -4.9921014723 & -4.9945990389 \\
\hline$|1,3,-3\rangle$ & $1 d_{5 / 2}$ & -4.9945990389 & -4.9921014723 & $|0,3,4\rangle$ & $0 g_{7 / 2}$ & -4.9945990389 & -4.9960022563 \\
\hline$|1,4,-4\rangle$ & $1 f_{7 / 2}$ & -4.9960022563 & -4.9945990389 & $|0,4,5\rangle$ & $0 h_{9 / 2}$ & -4.9960022563 & -4.9968759153 \\
\hline$|2,1,-1\rangle$ & $2 s_{1 / 2}$ & -4.9913874762 & -4.9863374401 & $|1,1,2\rangle$ & $1 d_{3 / 2}$ & -4.9913874762 & -4.9938803034 \\
\hline$|2,2,-2\rangle$ & $2 p_{3 / 2}$ & -4.9938803034 & -4.9913874762 & $|1,2,3\rangle$ & $1 f_{5 / 2}$ & -4.9938803034 & -4.9953083139 \\
\hline$|2,3,-3\rangle$ & $2 d_{5 / 2}$ & -4.9953083139 & -4.9938803034 & $|1,3,4\rangle$ & $1 g_{7 / 2}$ & -4.9953083139 & -4.9962128946 \\
\hline$|2,4,-4\rangle$ & $2 f_{7 / 2}$ & -4.9962128946 & -4.9953083139 & $|1,4,5\rangle$ & $1 h_{9 / 2}$ & -4.9962128946 & -4.9968280175 \\
\hline
\end{tabular}

4.1. Spin Symmetry Solution. Substituting (22) and (23) into (43) and defining a new variable of the form $s=e^{-\delta r}$, we get

$$
\begin{aligned}
& \frac{d^{2} F_{n \kappa}(s)}{d s^{2}}+\frac{1-s}{s(1-s)} \frac{d F_{n \kappa}(s)}{d s}+\frac{1}{\delta^{2} s^{2}(1-s)^{2}} \\
& \times\left[-\left(A+B+\beta^{2}\right) s^{2}+\left(B+2 \beta^{2}\right) s-\beta^{2}\right] \\
& \times F_{n \kappa}(s)=0 .
\end{aligned}
$$

Here

$$
\begin{gathered}
A=\lambda_{\kappa}\left(\lambda_{\kappa}+1\right) \delta^{2}+\gamma V_{1}, \\
B=-\gamma V_{0} .
\end{gathered}
$$

Comparing (45) with relations that are obtained in Section 3.1, we can easily obtain that

$$
\eta_{\kappa}=\sqrt{\frac{1}{4}+\frac{A}{\delta^{2}}}=\sqrt{\left(\kappa+U_{0 C}+\frac{1}{2}\right)^{2}+\frac{\gamma V_{1}}{\delta^{2}}} .
$$

We can obtain the energy eigenvalues of the Manning-Rosen potential with spin symmetric limit as

$$
\begin{gathered}
\left(n+\frac{1}{2}+\sqrt{\left(\kappa+U_{0 C}+\frac{1}{2}\right)^{2}+\frac{\gamma V_{1}}{\delta^{2}}}-\sqrt{\frac{\beta^{2}}{\delta^{2}}}\right)^{2} \\
=\frac{\gamma\left(V_{1}-V_{0}\right)+\beta^{2}}{\delta^{2}}+\left(\kappa+U_{0 C}+\frac{1}{2}\right)^{2}-\frac{1}{4}
\end{gathered}
$$

To find the corresponding wave functions, we can use (34), (35), and (36). Some numerical results of (48) are given in Table 5. In Table 5, we obtain numerical results in the spin symmetry in the presence and absence of tensor Coulomb form potential. We took a set of parameter values: $M=$ $5.0 \mathrm{fm}^{-1}, C_{s}=0, \delta=0.025 \mathrm{fm}^{-1}$, and $\alpha=0.01$. In Figure 6, we have investigated the effect of the tensor potential on the spin doublet splitting by considering the following pairs of orbital: $\left(1 p_{3 / 2}, 1 p_{1 / 2}\right),\left(1 f_{7 / 2}, 1 f_{5 / 2}\right)$. 


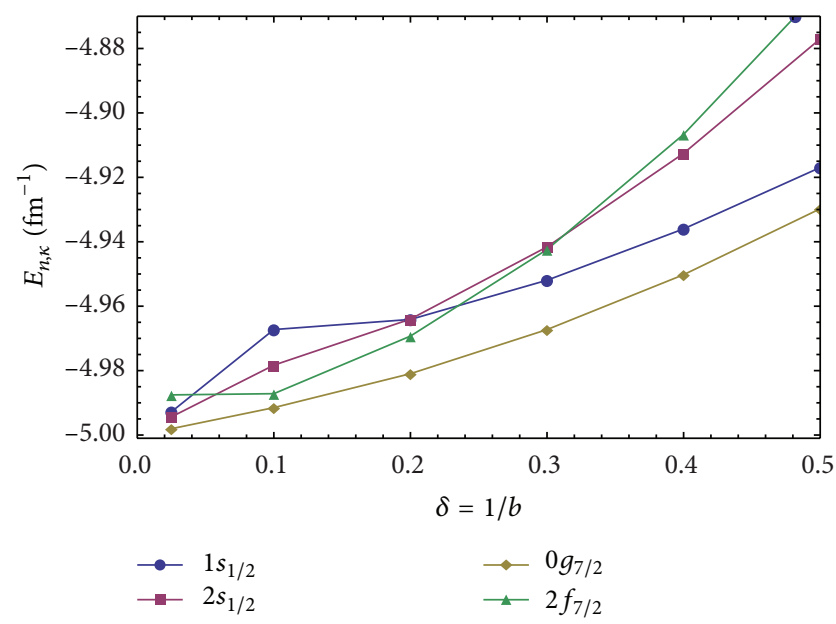

FIgURE 5: The variation of the energy spectrum $E_{n, k}$ for tensor Hulthén potential in the case of p-spin symmetry according to the screening parameter $\delta$. Here we selected $U_{0 H}=0.1, \alpha=0.01$.

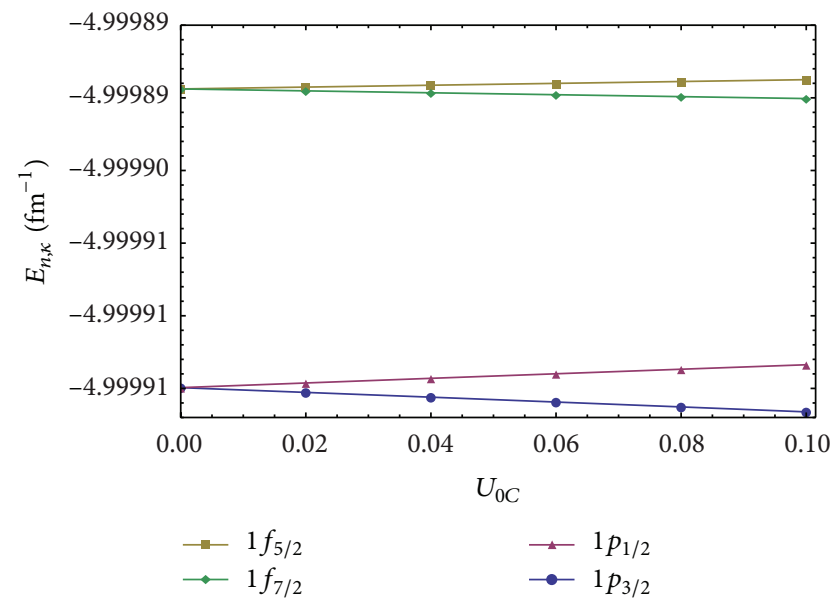

FIGURE 6: Contribution of the tensor Coulomb potential parameter to the energy levels in the case of spin symmetry.

4.2. p-Spin Symmetry Solution. Substituting (22) and (23) into (44) and defining a new variable of the form $s=e^{-\delta r}$, we get

$$
\begin{aligned}
& \frac{d^{2} G_{n \kappa}(s)}{d s^{2}}+\frac{1-s}{s(1-s)} \frac{d G_{n \kappa}(s)}{d s}+\frac{1}{\delta^{2} s^{2}(1-s)^{2}} \\
& \times\left[-\left(\widetilde{A}+\widetilde{B}+\widetilde{\beta}^{2}\right) s^{2}+\left(\widetilde{B}+2 \widetilde{\beta}^{2}\right) s-\widetilde{\beta}^{2}\right] \\
& \times G_{n \kappa}(s)=0 .
\end{aligned}
$$

Here

$$
\begin{gathered}
\widetilde{A}=\lambda_{\kappa}\left(\lambda_{\kappa}-1\right) \delta^{2}+\widetilde{\gamma} V_{1}, \\
\widetilde{B}=-\widetilde{\gamma} V_{0} .
\end{gathered}
$$

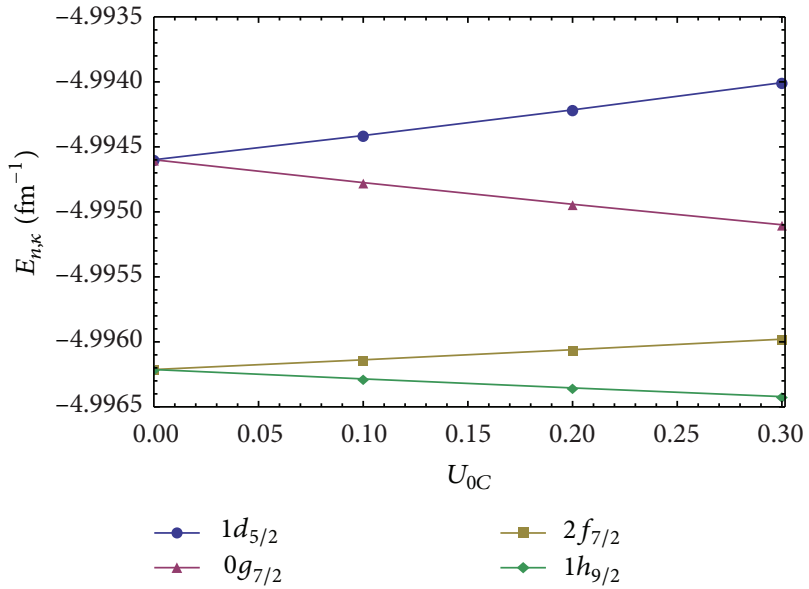

FIGURE 7: Contribution of the tensor Coulomb potential parameter to the energy levels in the case of p-spin symmetry.

Comparing (49) with relations that are obtained in Section 3.2, we can easily obtain that

$$
\widetilde{\eta}_{\kappa}=\sqrt{\frac{1}{4}+\frac{\widetilde{A}}{\delta^{2}}}=\sqrt{\left(\kappa+U_{0 C}-\frac{1}{2}\right)^{2}+\frac{\widetilde{\gamma} V_{1}}{\delta^{2}}} .
$$

We can obtain the energy eigenvalues of the Manning-Rosen potential with $\mathrm{p}$-spin symmetric limit as

$$
\begin{gathered}
\left(n+\frac{1}{2}+\sqrt{\left(\kappa+U_{0 C}-\frac{1}{2}\right)^{2}+\frac{\widetilde{\gamma} V_{1}}{\delta^{2}}}-\sqrt{\frac{\widetilde{\beta}^{2}}{\delta^{2}}}\right)^{2} \\
=\frac{\widetilde{\gamma}\left(V_{1}-V_{0}\right)+\widetilde{\beta}^{2}}{\delta^{2}}+\left(\kappa+U_{0 C}-\frac{1}{2}\right)^{2}-\frac{1}{4} .
\end{gathered}
$$

Also, we get the lower and upper component of the Dirac spinor as (41) and (42). In Table 6, we use the same parameters as in the previous subsection (with $C_{\mathrm{ps}}=0$ ) and we obtain numerical results in the p-spin symmetry in the presence and absence of tensor Coulomb form potential. In Figure 7, we have investigated the effect of the tensor potential on the pspin doublet splitting by considering some pairs of orbitals. Also the variation of the energy spectrum $\left(E_{n, \kappa}\right)$ of spin and p-spin symmetry given by Tables 5 and 6 , according to the screening parameter $\delta$ for some states, is shown in Figures 8 and 9.

\section{Conclusion}

In this paper, we obtained the approximate analytical solutions of the Dirac equation for the Manning-Rosen potential with the consideration of Hulthén and Coulomb-like potentials as tensor interaction within the framework of spin and pseudospin symmetry limits using the generalized parametric NU technique. We have obtained the energy levels in a closed form and the corresponding wave functions in terms of the Jacobi polynomials. Also we obtained the energy eigenvalues in the absence $\left(U_{0 H}=0\right)$ and the presence 


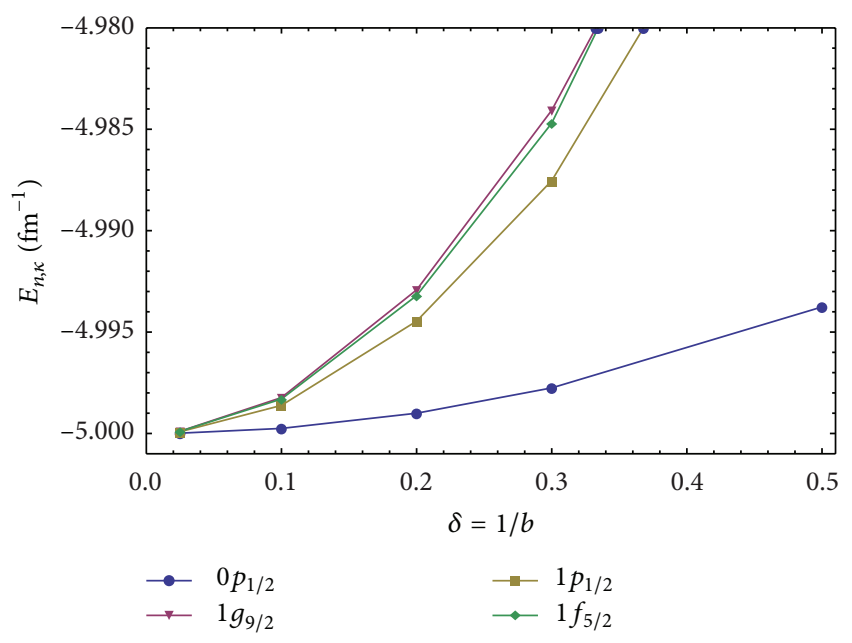

FIgURE 8: The variation of the energy spectrum $E_{n, k}$ for tensor Coulomb potential in the case of spin symmetry according to the screening parameter $\delta$. Here we selected $U_{0 C}=0.1, \alpha=0.01$.

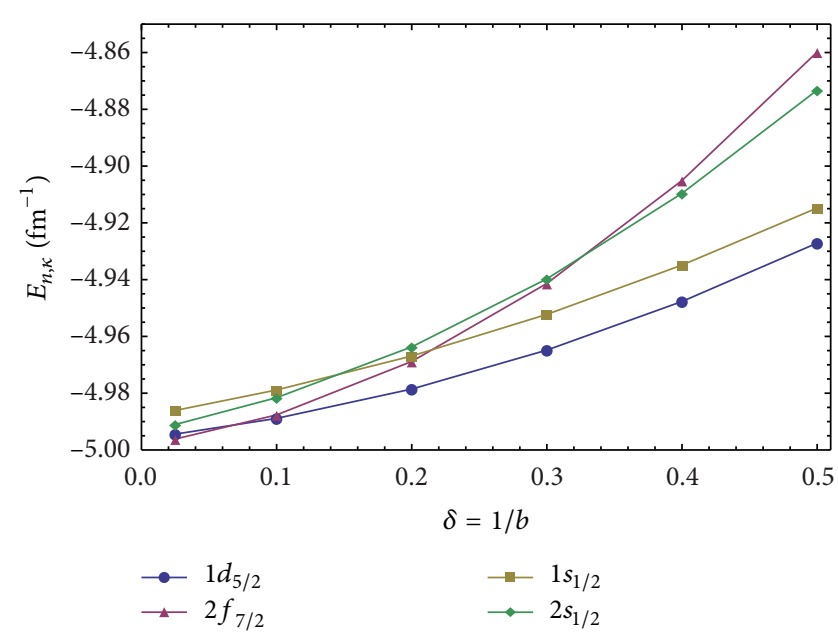

FIGURE 9: The variation of the energy spectrum $E_{n, k}$ for tensor Coulomb potential in the case of $\mathrm{p}$-spin symmetry according to the screening parameter $\delta$. Here we selected $U_{0 C}=0.1, \alpha=0.01$.

$\left(U_{0 H}=0.1,0.2\right)$ of the Hulthén tensor interaction and the energy eigenvalues in the absence $\left(U_{0 C}=0\right)$ and the presence $\left(U_{0 C}=1\right)$ of the Coulomb-like tensor interaction for various values of the quantum numbers $n$ and $\kappa$. The results are reported in Tables 3-6 under the condition of the spin and pseudospin symmetries, respectively, where we can see the way the tensor interaction affects the degeneracy of the system. Figures 2, 3, 6, and 7 present the effect of the Hulthén and Coulomb-like tensor interaction on the bound states, respectively. Obviously, the degeneracy between the members of doublet states in spin and pseudospin symmetries is removed by these tensor interaction potentials. We represent the effects of the screening parameter $\delta$ on the bound states for Hulthén and Coulomb-like tensor interaction in Figures $4,5,8$, and 9 , respectively. It is seen that if the $\delta$ parameter increases, for both tensor interactions, the bound states become more bounded in both for symmetry limits. Therefore, the results of our work find many applications in both nuclear and Hadron physics and they obviously indicate the degeneracy-removing role of tensor interaction [104], which plays a significant role in the spectroscopes.

\section{Conflict of Interests}

The authors declare that there is no conflict of interests regarding the publication of this paper.

\section{Acknowledgments}

The authors highly appreciate the very constructive comments and suggestions provided from the kind referees and editors.

\section{References}

[1] A. Arima, M. Harvey, and K. Shimizu, "Pseudo LS coupling and pseudo SU3 coupling schemes," Physics Letters B, vol. 30, no. 8, pp. 517-522, 1969.

[2] K. T. Hecht and A. Adler, "Generalized seniority for favored $J \neq$ 0 pairs in mixed configurations," Nuclear Physics A, vol. 137, no. 1, pp. 129-143, 1969.

[3] A. Bohr, I. Hamamoto, and B. R. Mottelson, "Pseudospin in rotating nuclear potentials," Physica Scripta, vol. 26, pp. 267$272,1982$.

[4] J. Dudek, W. Nazarewicz, Z. Szymanski, and G. A. Leander, "Abundance and systematics of nuclear superdeformed states; relation to the pseudospin and pseudo-SU(3) symmetries," Physical Review Letters, vol. 59, no. 13, pp. 1405-1408, 1987.

[5] D. Troltenier, C. Bahri, and J. P. Draayer, "Generalized pseudoSU(3) model and pairing," Nuclear Physics A, vol. 586, no. 1, pp. 53-72, 1995.

[6] J. N. Ginocchio, "U(3) and Pseudo-U(3) symmetry of the relativistic harmonic oscillator," Physical Review Letters, vol. 95, no. 25, Article ID 252501, 3 pages, 2005.

[7] J. N. Ginocchio, "Pseudospin as a relativistic symmetry," Physical Review Letters, vol. 78, no. 3, pp. 436-439, 1997.

[8] J. Meng, K. Sugawara-Tanabe, S. Yamaji, P. Ring, and A. Arima, "Pseudospin symmetry in relativistic mean field theory," Physical Review C, vol. 58, no. 2, pp. R628-R631, 1998.

[9] A. D. Alhaidari, H. Bahlouli, and A. Al-Hasan, "Dirac and Klein-Gordon equations with equal scalar and vector potentials," Physics Letters A, vol. 349, no. 1-4, pp. 87-97, 2006.

[10] G.-F. Wei and S.-H. Dong, "Approximately analytical solutions of the Manning-Rosen potential with the spin-orbit coupling term and spin symmetry," Physics Letters A, vol. 373, no. 1, pp. 49-53, 2008.

[11] G.-F. Wei and S.-H. Dong, "The spin symmetry for deformed generalized Pöschl-Teller potential," Physics Letters A, vol. 373, no. 29, pp. 2428-2431, 2009.

[12] G.-F. Wei and S.-H. Dong, "A novel algebraic approach to spin symmetry for Dirac equation with scalar and vector second Pöschl-Teller potentials," European Physical Journal A, vol. 43, no. 2, pp. 185-190, 2010.

[13] Gao-Feng Wei and Shi-Hai Dong, "Spin symmetry in the relativistic symmetrical well potential including a proper approximation to the spin-orbit coupling term," Physica Scripta, vol. 81, Article ID 035009, 2010. 
[14] S. M. Ikhdair and R. Sever, "Approximate bound state solutions of Dirac equation with Hulthén potential including Coulomblike tensor potential," Applied Mathematics and Computation, vol. 216, no. 3, pp. 911-923, 2010.

[15] C. Berkdemir, "Pseudospin symmetry in the relativistic Morse potential including the spin-orbit coupling term," Nuclear Physics A, vol. 770, no. 1-2, pp. 32-39, 2006.

[16] G.-F. Wei and S.-H. Dong, "Algebraic approach to pseudospin symmetry for the Dirac equation with scalar and vector modified Pöschl-teller potentials," EPL, vol. 87, no. 4, Article ID 40004, 2009.

[17] G.-F. Wei and S.-H. Dong, "Pseudospin symmetry in the relativistic Manning-Rosen potential including a Pekeris-type approximation to the pseudo-centrifugal term," Physics Letters $B$, vol. 686, no. 4-5, pp. 288-292, 2010.

[18] G.-F. Wei and S.-H. Dong, "Pseudospin symmetry for modified Rosen-Morse potential including a Pekeris-type approximation to the pseudo-centrifugal term," European Physical Journal A, vol. 46, no. 2, pp. 207-212, 2010.

[19] C.-S. Jia, P. Guo, and X.-L. Peng, "Exact solution of the DiracEckart problem with spin and pseudospin symmetry," Journal of Physics A, vol. 39, no. 24, article 7737, 2006.

[20] Y. Xu, S. He, and C.-S. Jia, "Approximate analytical solutions of the Dirac equation with the Pöschl-Teller potential including the spin-orbit coupling term," Journal of Physics A, vol. 41, no. 25, Article ID 255302, 2008.

[21] S.-H. Dong and J. García-Ravelo, "Exact solutions of the $s$-wave Schrödinger equation with Manning-Rosen potential," Physica Scripta, vol. 75, no. 3, article 307, 2007.

[22] C.-Y. Chen, F.-L. Lu, and D.-S. Sun, "Exact solutions of scattering states for the $s$-wave Schrödinger equation with the Manning-Rosen potential," Physica Scripta, vol. 76, no. 5, pp. 428-430, 2007.

[23] A. Diaf, A. Chouchaoui, and R. J. Lombard, "Feynman integral treatment of the Bargmann potential," Annals of Physics, vol. 317, no. 2, pp. 354-365, 2005.

[24] S. M. Ikhdair and R. Sever, "Approximate $l$-state solutions of the $D$-dimensional Schrödinger equation for Manning-Rosen potential," Annalen der Physik, vol. 17, no. 11, pp. 897-910, 2008.

[25] X.-Y. Gu and S.-H. Dong, "Energy spectrum of the ManningRosen potential including centrifugal term solved by exact and proper quantization rules," Journal of Mathematical Chemistry, vol. 49, no. 9, pp. 2053-2062, 2011.

[26] W.-C. Qiang and S.-H. Dong, "Analytical approximations to the solutions of the Manning-Rosen potential with centrifugal term," Physics Letters A, vol. 368, no. 1-2, pp. 13-17, 2007.

[27] G.-F. Wei, C.-Y. Long, and S.-H. Dong, "The scattering of the Manning-Rosen potential with centrifugal term," Physics Letters $A$, vol. 372, no. 15, pp. 2592-2596, 2008.

[28] G.-F. Wei, Z.-Z. Zhen, and S.-H. Dong, “The relativistic bound and scattering states of the Manning-Rosen potential with an improved new approximate scheme to the centrifugal term," Central European Journal of Physics, vol. 7, no. 1, pp. 175-183, 2009.

[29] Q. Wen-Chao and D. Shi-Hai, "The Manning-Rosen potential studied by a new approximate scheme to the centrifugal term," Physica Scripta, vol. 79, Article ID 045004, 2009.

[30] M. Moshinsky and A. Szczepaniak, "The Dirac oscillator," Journal of Physics A, vol. 22, no. 17, pp. L817-L819, 1989.

[31] V. I. Kukulin, G. Loyola, and M. Moshinsky, "A Dirac equation with an oscillator potential and spin-orbit coupling," Physics Letters A, vol. 158, no. 1-2, pp. 19-22, 1991.
[32] R. Lisboa, M. Malheiro, A. S. De Castro, P. Alberto, and M. Fiolhais, "Pseudospin symmetry and the relativistic harmonic oscillator," Physical Review C, vol. 69, no. 2, Article ID 024319, 2004.

[33] P. Alberto, R. Lisboa, M. Malheiro, and A. S. De Castro, “Tensor coupling and pseudospin symmetry in nuclei," Physical Review C, vol. 71, no. 3, Article ID 034313, 2005.

[34] H. Akcay, "Dirac equation with scalar and vector quadratic potentials and Coulomb-like tensor potential," Physics Letters A, vol. 373, no. 6, pp. 616-620, 2009.

[35] H. Akcay, "The Dirac oscillator with a Coulomb-like tensor potential," Journal of Physics A, vol. 40, no. 24, article 6427, 2007.

[36] O. Aydoğdu and R. Sever, "Exact pseudospin symmetric solution of the dirac equation for pseudoharmonic potential in the presence of tensor potential," Few-Body Systems, vol. 47, no. 3, pp. 193-200, 2010.

[37] O. Aydoğdu and R. Sever, "Pseudospin and spin symmetry in the Dirac equation with Woods-Saxon potential and tensor potential," European Physical Journal A, vol. 43, no. 1, pp. 73-81, 2009.

[38] M. Hamzavi, A. A. Rajabi, and H. Hassanabadi, "Exact pseudospin symmetry solution of the Dirac equation for spatiallydependent mass Coulomb potential including a Coulomb-like tensor interaction via asymptotic iteration method," Physics Letters A, vol. 374, no. 42, pp. 4303-4307, 2010.

[39] M. Hamzavi, A. A. Rajabi, and H. Hassanabadi, "Exactly complete solutions of the Dirac equation with pseudoharmonic potential including linear plus Coulomb-like tensor potential," International Journal of Modern Physics A, vol. 26, no. 7-8, pp. 1363-1374, 2011.

[40] S. M. Ikhdair, "An improved approximation scheme for the centrifugal term and the Hulthén potential," European Physical Journal A, vol. 39, no. 3, pp. 307-314, 2009.

[41] C. S. Lam and Y. P. Varshni, "Energies of s eigenstates in a static screened coulomb potential," Physical Review A, vol. 4, no. 5, pp. 1875-1881, 1971.

[42] B. Durand and L. Durand, "Duality for heavy-quark systems," Physical Review D, vol. 23, no. 5, pp. 1092-1102, 1981.

[43] R. L. Hall, "Envelope representations for screened Coulomb potentials," Physical Review A, vol. 32, no. 1, pp. 14-18, 1985.

[44] R. Dutt, K. Chowdhury, and Y. P. Varshni, "An improved calculation for screened Coulomb potentials in RayleighSchrödinger perturbation theory," Journal of Physics A, vol. 18, no. 9, article 1379, 1985.

[45] J. Lindhard and P. G. Hansen, "Atomic effects in low-energy beta decay: the case of tritium," Physical Review Letters, vol. 57, no. 8, pp. 965-967, 1986.

[46] I. S. Bitensky, V. K. Ferleger, and I. A. Wojciechowski, "Distortion of $\mathrm{H} 2$ potentials by embedding into an electron gas at molecule scattering by a metal surface," Nuclear Instruments and Methods in Physics Research B, vol. 125, no. 1-4, pp. 201-206, 1997.

[47] C.-S. Jia, J.-Y. Wang, S. He, and L.-T. Sun, "Shape invariance and the supersymmetry WKB approximation for a diatomic molecule potential," Journal of Physics A, vol. 33, no. 39, pp. 6993-6998, 2000.

[48] J. A. Olson and D. A. Micha, "Transition operators for atomatom potentials: the Hilbert-Schmidt expansion," The Journal of Chemical Physics, vol. 68, no. 10, pp. 4352-4356, 1978.

[49] G. R. Satchler, Direct Nuclear Reactions, Oxford University Press, London, UK, 1983. 
[50] B. Roy and R. Roychoudhury, "Dirac equation with Hulthen potential: an algebraic approach," Journal of Physics A, vol. 23, no. 21, article 5095, 1990.

[51] E. D. Filho and R. M. Ricotta, "Suppersymmetry, variational method and Hulthén potential," Modern Physics Letters A, vol. 10, pp. 1613-1618, 1995.

[52] S.-W. Qian, B.-W. Huang, and Z.-Y. Gu, "Supersymmetry and shape invariance of the effective screened potential," New Journal of Physics, vol. 4, pp. 13.1-13.6, 2002.

[53] H. Ciftci, R. L. Hall, and N. Saad, "Asymptotic iteration method for eigenvalue problems," Journal of Physics A, vol. 36, no. 47, pp. 11807-11816, 2003.

[54] A. Soylu, O. Bayrak, and I. Boztosun, "An approximate solution of Dirac-Hulthén problem with pseudospin and spin symmetry for any $\kappa$ state," Journal of Mathematical Physics, vol. 48, no. 8, Article ID 082302, 2007.

[55] S. M. Ikhdair, C. Berkdemir, and R. Sever, "Spin and pseudospin symmetry along with orbital dependency of the Dirac-Hulthén problem," Applied Mathematics and Computation, vol. 217, no. 22, pp. 9019-9032, 2011.

[56] L. C. Biedenharn, "Remarks on the relativistic Kepler problem," vol. 126, pp. 845-851, 1962.

[57] S. Haouat and L. Chetouani, "Approximate solutions of KleinGordon and Dirac equations in the presence of the Hulthén potential," Physica Scripta, vol. 77, no. 2, Article ID 025005, 2008.

[58] M.-C. Zhang, "Exact bound state solutions of the Klein-Gordon particle in Hulthén potential," Chinese Physics B, vol. 17, no. 9, pp. 3214-3216, 2008.

[59] Y. Chargui, L. Chetouani, and A. Trabelsi, "Exact solution of the one-dimensional Klein-Gordon equation with scalar and vector linear potentials in the presence of a minimal length," Chinese Physics B, vol. 19, no. 2, Article ID 020305, 2010.

[60] Y. Zhou and J.-Y. Guo, "The relativistic bound states for a new ring-shaped harmonic oscillator," Chinese Physics B, vol. 17, no. 2, pp. 380-384, 2008.

[61] X.-X. Zeng and Q. Li, "On particles tunneling from the TaubNUT-AdS black hole," Chinese Physics B, vol. 18, no. 11, pp. 47164720, 2009.

[62] G.-J. Ni, J.-J. Xu, and S.-Y. Lou, "Reduced Dirac equation and Lamb shift as off-mass-shell effect in quantum electrodynamics," Chinese Physics B, vol. 20, no. 2, Article ID 020302, 2011.

[63] H.-L. Li, "Fermion tunneling from squashed black holes in the Gödel universe and charged Kaluza - Klein space - Time," Chinese Physics B, vol. 20, no. 3, Article ID 030402, 2011.

[64] F. Taşkın and G. Koçak, "Spin symmetric solutions of Dirac equation with Pöschl-Teller potential," Chinese Physics B, vol. 20, Article ID 070302, 2011.

[65] S.-Q. Zhou, "Bridge density functional approximation for nonuniform hard core repulsive Yukawa fluid," Chinese Physics $B$, vol. 17, no. 10, pp. 3812-3821, 2008.

[66] Z. Y. Ma and S. H. Ma, "Analytical solutions and rogue waves in (3+1)-dimensional nonlinear Schrödinger equation," Chinese Physics B, vol. 21, Article ID 030507, 2012.

[67] C. Y. Chen, F. L. Lu, and Y. You, "Scattering states of modified Pöschlben-Teller potential in D-dimension," Chinese Physics B, vol. 21, Article ID 030302, 2012.

[68] M. Hamzavi, M. Eshghi, and S. M. Ikhdair, "Effect of tensor interaction in the Dirac-attractive radial problem under pseudospin symmetry limit," Journal of Mathematical Physics, vol. 53, Article ID 082101, 2012.
[69] F. L. Lu and C. Y. Chen, "Bound states of the Schrödinger equation for the Pöschl-Teller double-ring-shaped Coulomb potential," Chinese Physics B, vol. 19, no. 10, Article ID 100309, 2010.

[70] F. Taşkın and G. Koçak, "Approximate solutions of Schrödinger equation for Eckart potential with centrifugal term," Chinese Physics B, vol. 19, no. 9, Article ID 090314, 2010.

[71] G. Wei and W.-L. Chen, "Continuum states of modified Morse potential," Chinese Physics B, vol. 19, no. 9, Article ID 090308, 2010.

[72] G.-H. Sun and S.-H. Dong, "Relativistic treatment of spinless particles subject to a Tietz-Wei oscillator," Communications in Theoretical Physics, vol. 58, no. 2, pp. 195-197, 2012.

[73] M. Koçak and B. Gönül, "A search on dirac equation," Chinese Physics Letters, vol. 24, no. 11, pp. 3024-3027, 2007.

[74] K.-L. Su, "The most general corrdinate transformation between the hydrogen atom and harmonic oscillator in arbitrary dimensions," Chinese Physics Letters, vol. 14, no. 10, pp. 721-723, 1997.

[75] G.-F. Wei, C.-Y. Long, S.-J. Qin, and X. Zhang, "Analytical approximations to the arbitrary l-wave bound state solutions of the Klein-Gordon equation for the Manning-Rosen potential," Acta Physica Sinica, vol. 57, no. 11, pp. 6730-6735, 2008.

[76] M.-C. Zhang and Z.-B. Wang, "Bound state solutions of relativistic particles in a new ring-shaped non-harmonic oscillator potential," Acta Physica Sinica, vol. 56, no. 7, pp. 3688-3692, 2007.

[77] M. C. Zhang and Z. B. Wang, "Bound states of relativistic particles in the second Pöschl-Teller potentials," Acta Physica Sinica, vol. 55, pp. 525-528, 2006.

[78] M. C. Zhang and Z. B. Wang, "Bound states of the Klein-Gordon equation and Dirac equation with the Manning-Rosen scalar and vector potentials," Acta Physica Sinica, vol. 55, pp. 521-524, 2006.

[79] F. L. Lu and C. Y. Chen, "Bound states of Klein-Gordon equation for ring-shaped non-spherical harmonic oscillator potentials," Acta Physica Sinica, vol. 53, pp. 1652-1656, 2004.

[80] G. Chen, "Bound states of relativistic particles in Rosen-Morse potential," Acta Physica Sinica, vol. 53, no. 3, pp. 684-687, 2004.

[81] W.-C. Qiang, "Bound states of the Klein-Gordon and Dirac equations for scalar and vector harmonic oscillator potentials," Chinese Physics, vol. 11, no. 8, pp. 757-759, 2002.

[82] H.-M. Li, "Mapping deformation method and its application to nonlinear equations," Chinese Physics, vol. 11, no. 11, pp. 1111-1114, 2002.

[83] H.-Y. Fan and M.-Z. Sun, "Angular velocity and coriolis effect in time-dependent quantum mechanical $\mathrm{SU}_{2}$ rotation," Chinese Physics B, vol. 10, no. 5, pp. 380-383, 2001.

[84] G.-L. Li, R.-H. Yue, K.-J. Shi, and B.-Y. Hou, "Exact solution for extended Essler-Korepin-Schoutens model with open boundary conditions," Chinese Physics B, vol. 10, no. 2, pp. 113-116, 2001.

[85] H.-X. Lu, X.-Q. Wang, and Y.-D. Zhang, "Exact solution for Super-Jaynes-cummings model," Chinese Physics B, vol. 9, no. 5, pp. 325-328, 2000.

[86] H. Jing and J.-S. Wu, “Time-dependent Landau system and nonadiabatic Berry phase in two dimensions," Chinese Physics $B$, vol. 9, no. 7, 2000.

[87] C. Y. Chen, "General formula and recurrence formula for radial matrix elements of $n$-dimensional isotropic harmonic oscillator," Chinese Physics B, vol. 9, pp. 731-736, 2000. 
[88] H.-X. Lu, K.-J. Liu, J.-W. Pan, and Y.-D. Zhang, "Energy spectrum and wave function for multi-mode coupled nonidentical harmonic oscillators," Chinese Physics B, vol. 9, no. 1, pp. 5-8, 2000.

[89] W.-C. Qiang, "Bound states of the Klein-Gordon and Dirac equations for potential $V(r)=A r^{-2}-B r^{-1}$," Chinese Physics, vol. 12, no. 10, pp. 1054-1057, 2003.

[90] Z.-X. Ni, "Vector ladder operators for the central potentials," Chinese Physics B, vol. 8, no. 1, pp. 8-13, 1999.

[91] S.-G. Zhou, J. Meng, and P. Ring, "Spin symmetry in the antinucleon spectrum," Physical Review Letters, vol. 91, no. 26, Article ID 262501, 4 pages, 2003.

[92] X. T. He, S. G. Zhou, J. Meng, E. G. Zhao, and W. Scheid, "Test of spin symmetry in anti-nucleon spectra," The European Physical Journal A, vol. 28, no. 3, pp. 265-269, 2006.

[93] C.-Y. Song, J.-M. Yao, and J. Meng, "Spin symmetry for antilambda spectrum in atomic nucleus," Chinese Physics Letters, vol. 26, no. 12, Article ID 122102, 2009.

[94] C.-Y. Song and J.-M. Yao, "Polarization effect on the spin symmetry for anti-Lambda spectrum in ${ }^{16} \mathrm{O}+\bar{\lambda}$ system," Chinese Physics C, vol. 34, no. 9, pp. 1425-1427, 2010.

[95] J. N. Ginocchio, "The relativistic foundations of pseudospin symmetry in nuclei," Nuclear Physics A, vol. 654, no. 1, pp. 663c668c, 1999.

[96] J. N. Ginocchio, "A relativistic symmetry in nuclei," Physics Report, vol. 315, no. 1-4, pp. 231-240, 1999.

[97] J. Meng, K. Sugawara-Tanabe, S. Yamaji, and A. Arima, "Pseudospin symmetry in $\mathrm{Zr}$ and $\mathrm{Sn}$ isotopes from the proton drip line to the neutron drip line," Physical Review C, vol. 59, no. 1, pp. 154-163, 1999.

[98] M. G. Miranda, G.-H. Sun, and S.-H. Dong, "The solution of the second pöschlteller like potential by Nikiforov-Uvarov method," International Journal of Modern Physics E, vol. 19, no. 1, pp. 123-129, 2010.

[99] M.-C. Zhang, G.-H. Sun, and S.-H. Dong, "Exactly complete solutions of the Schrödinger equation with a spherically harmonic oscillatory ring-shaped potential," Physics Letters A, vol. 374, no. 5, pp. 704-708, 2010.

[100] G. Szegö, Orthogonal Polynomials, American Mathematical Society, New York, NY, USA, 1939.

[101] C. Tezcan and R. Sever, "A general approach for the exact solution of the Schrödinger equation," International Journal of Theoretical Physics, vol. 48, no. 2, pp. 337-350, 2009.

[102] A. F. Nikiforov and V. B. Uvarov, Special Functions of Mathematical Physics, Birkhäuser, Basel, Switzerland, 1988.

[103] S. M. Ikhdair, "Rotational and vibrational diatomic molecule in the Klein-Gordon equation with hyperbolic scalar and vector potentials," International Journal of Modern Physics C, vol. 20, no. 10, pp. 1563-1582, 2009.

[104] J. N. Ginocchio, "Relativistic symmetries in nuclei and hadrons," Physics Reports, vol. 414, no. 4-5, pp. 165-261, 2005. 

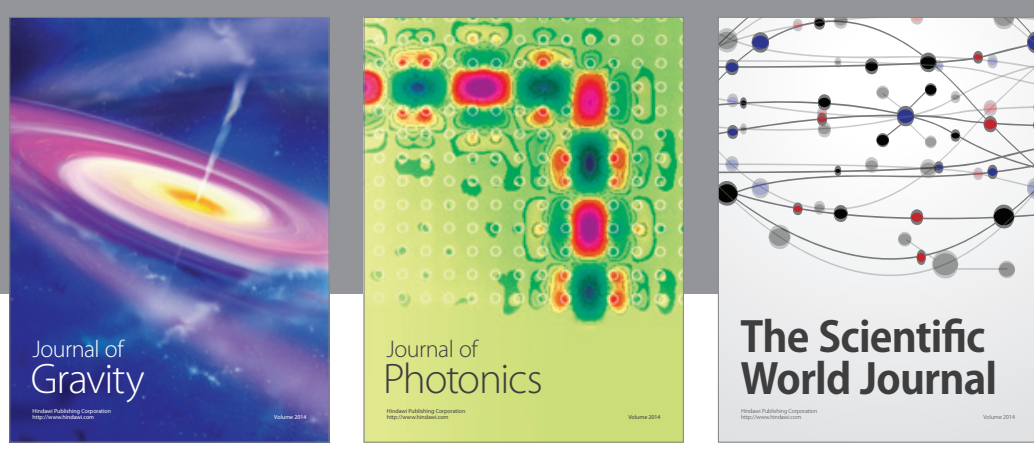

The Scientific World Journal
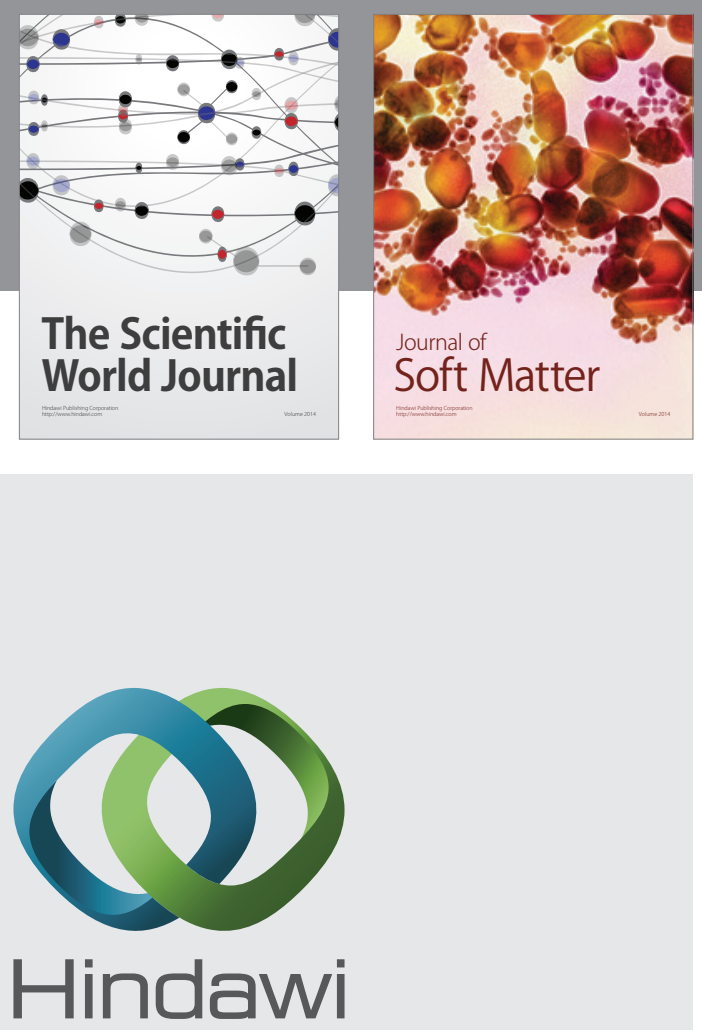

Submit your manuscripts at

http://www.hindawi.com

nternational Journal of

Statistical Mechanics
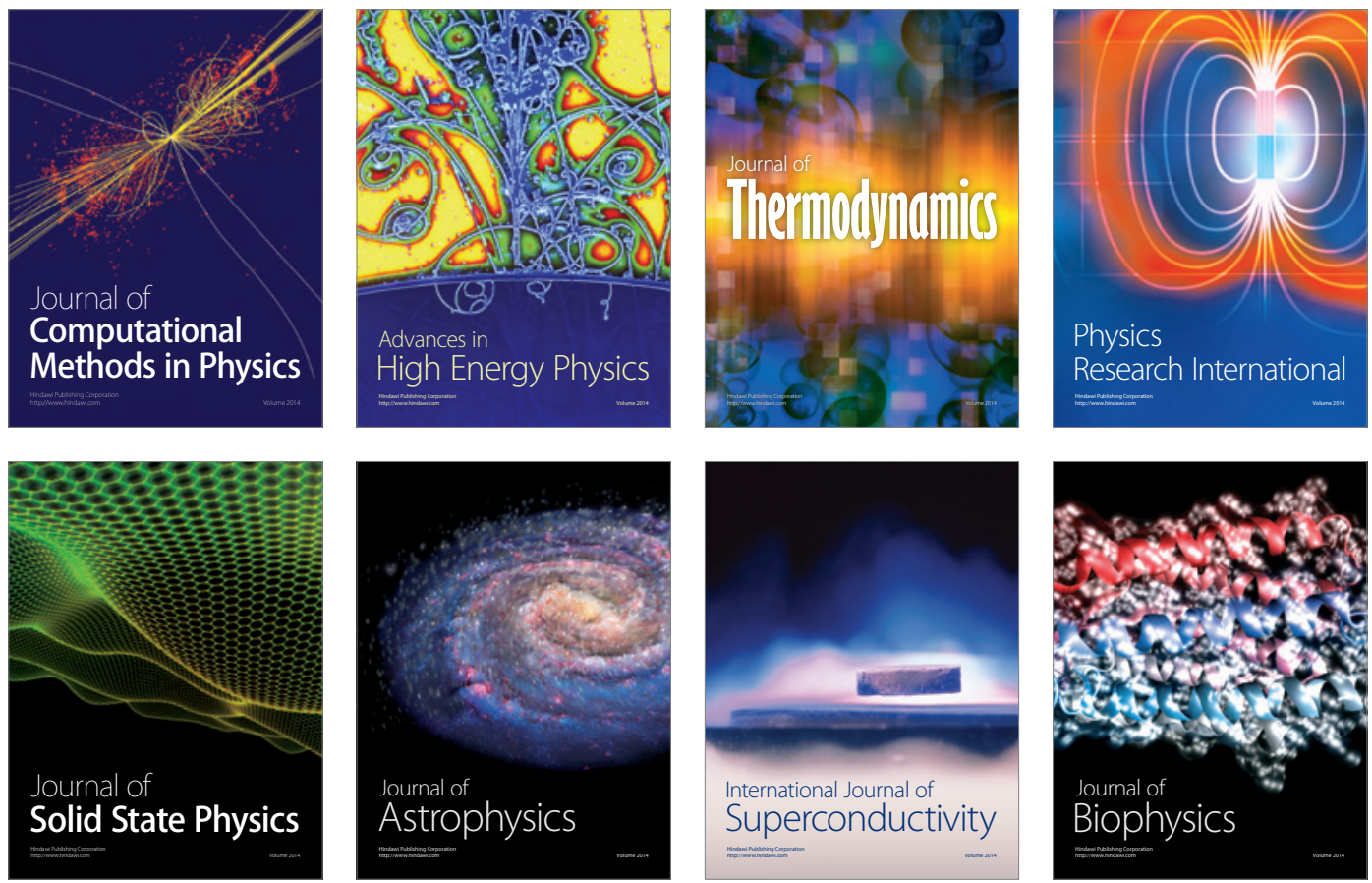
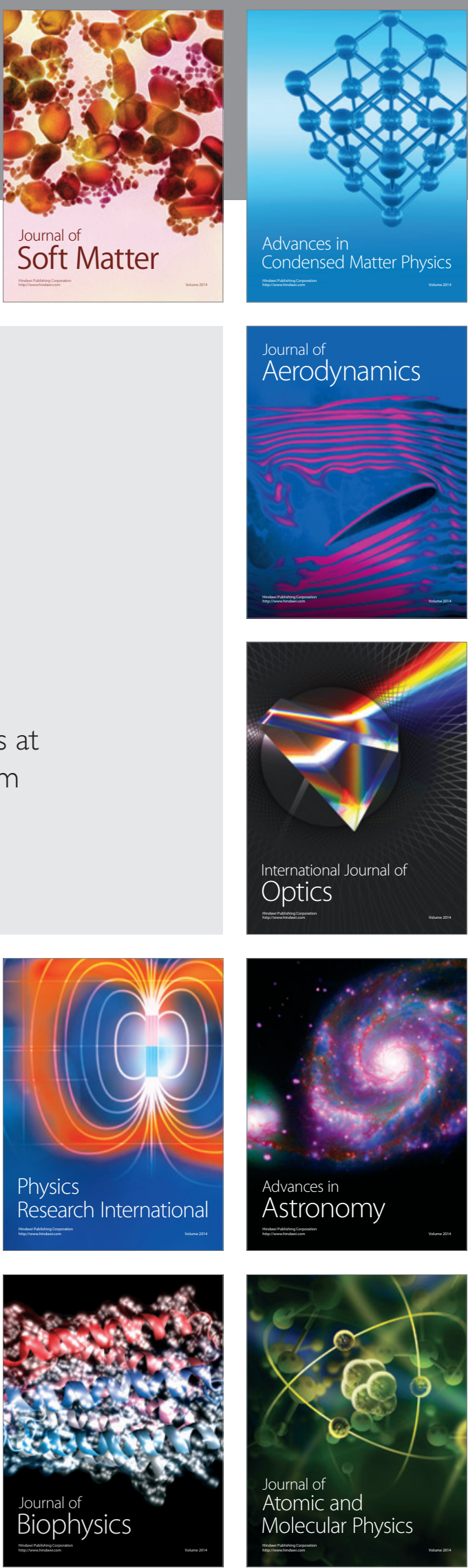